\title{
Correlations between nebular emission and the continuum spectral shape in SDSS galaxies
}

\author{
Zsuzsanna Győry ${ }^{1,2}$, Alexander S. Szalay ${ }^{3,1}$, Tamás Budavári ${ }^{3}$, István Csabai ${ }^{1,3}$, Stéphane \\ Charlot $^{4,5}$ \\ gyory@complex.elte.hu
}

\begin{abstract}
We present a statistical study of correlations and dimensionality of emission lines carried out on a sample of over 40,000 SDSS galaxies. Using principal component analysis we found that the equivalent widths of the 11 strongest lines can be well represented using three parameters. We also explore correlations of emission pattern with the eigenspace representation of the continuum spectrum. The observed relations are used to provide an empirical prescription for expectation values and variances of emission line strengths as a function of spectral shape. We show that this estimation of emission lines has a sufficient accuracy to make it suitable for photometric applications. The method has already proved useful in photometric redshift estimation
\end{abstract}

Subject headings: galaxies: evolution — methods: statistical — techniques: spectroscopic

\section{Introduction}

Galaxy spectral models play an essential role in the interpretation of observational data. It is important to be able to characterize models with few parameters as accurately as possible especially when working with photometric measurements. Our motivation for exploring the dimensionality of the emission pattern and its correlation with the continuum spectrum originates in photometric redshift estimation. There are basically two different approaches to determining redshifts of galaxies from their multi-band photometric data. One is empirical, for example via fitting the colorredshift relation (Connolly et al. (1995)), nearest neighbors (Budavári (2001)), Kd-tree (Csabai

\footnotetext{
${ }^{1}$ Department of Physics, Eötvös University, Budapest, Pf. 32, H-1518, Hungary

${ }^{2}$ Max Planck Institute for Astronomy, Königstuhl 17, 69117 Heidelberg, Germany

${ }^{3}$ Department of Physics and Astronomy, The Johns Hopkins University, 3701 San Martin Drive, Baltimore, MD 21218

${ }^{4}$ Max-Planck-Institute für Astrophysik, Karl-Schwarzschild-Strasse 1, 85748 Garching, Germany

${ }^{5}$ Institut d'Astrophysique de Paris, CNRS, 98 bis boulevard Arago, F-75014 Paris, France
} 
et al. (2003)) or artificial neural networks (Firth et al. (2003)). The other approach is based on template spectra. The templates might be either empirical, like Coleman, Wu, \& Weedman (1980), or synthetic, e.g., Bruzual \& Charlot (2003) and Fioc \& Rocca-Volmerange (1997). The templates are redshifted and convolved with the filter curves of the survey. The simulated fluxes serve as a reference set to match with the photometry of the real objects. The best match gives an estimate of the redshift and spectral type of a galaxy. (For an overview, see, e.g., Csabai et al. (2003).) In many redshift studies synthetic spectra are used, for example in COMBO-17 (Wolf et al. (2004)), Hyperz (Bolzonella et al. (2000)), and EAZY (Brammer et al. (2008)). Modern surveys with good signal-to-noise ratio $(\mathrm{S} / \mathrm{N})$ and resolution, such as the co-added stripe 82 of the Sloan Digital Sky Survey (SDSS), PanSTARRS (Kaiser et al. (2005)), or LSST (Tyson et al. (2002)) make even a more general photometric parameter inversion realistic Budavári (2009). Beyond redshift, one may address other physical parameters as well, such as star formation history, metallicity, or dust. Then an ensemble of model spectra parameterized by a set of such observables is the best starting point for calibrating the photometric inversion procedure. Not all quantities of interest will be unambiguously visible from the photometric data, but their degeneracies, correlations and errors can be well assessed by the calibration. Knowing the propagation of the uncertainties allows one to minimize the error of a particular observable and thus optimize the entire method. This concept assumes realistic model templates. The templates should account for all features contributing to the integrated fluxes, i.e., the spectral continua as well as the strongest emission lines.

A suitable spectral model should include the radiation of stars, ionized gas, and the effect of dust. There are works by Stasinska \& Leitherer (1996); Moy et al. (2001); Charlot \& Longhetti (2001); Panuzzo et al. (2003) that couple these components. Stellar continua are usually modeled using population synthesis models, e.g. Bruzual \& Charlot (2003), PEGASE (Fioc \& RoccaVolmerange 1997). Emission lines in star forming (SF) H II regions are generated by photoionization codes, e.g. PHOTO (Stasinska 1990), CLOUDY (Ferland 1996). In general, a particular model is defined by age, metallicity, star formation history and initial mass function of the underlying stellar population. Furthermore chemical composition, density and geometry of the ionized gas as well as dust content and certain characteristics of dust also define a model. In order to reduce the number of free parameters one usually applies simplifying assumptions and self-consistency constraints, i.e. empirical relations between the physical quantities. This enables one to produce models described with a reasonable accuracy with about three stellar and three gas parameters.

Principal component analysis (PCA) has proved to be a powerful tool in exploring the correlations between emission line properties and continuum spectral characteristics. Sodré \& Stasinska (1999) and Stasinska \& Sodré (2001) used it for statistical analysis of spectral features of nearby spiral galaxies. They identified the trends of emission line equivalent widths (EWs) as a function of spectral type obtained by PCA.

SDSS provides data suitable for statistical analyses of nebular emission on a large sample of galaxies. There have been numerous studies addressing the physical properties of SDSS emission line galaxies (Brinchmann et al. (2004), Kauffmann et al. (2003), Tremonti et al. (2004)). The aim 
of our present study is to elucidate the statistical description of galaxy emission lines. This is complementary to the previous studies as it allows one to explore, for example, the contamination of photometric magnitudes by emission lines in an efficient way.

In this paper we perform PCA on emission line EWs of SDSS galaxies in order to find the minimal number of independent parameters describing the emission line pattern with a reasonable accuracy. We then explore their correlations with the continuum spectrum PCA parameters and determine the most probable emission line pattern and its variations as a function of the emissionfree continuum spectrum. These relations enable us to add emission lines to population synthesis model spectra in an empirical way. This prescription was used to improve the empirical spectral templates used in SDSS photometric redshift estimation.

\section{Data}

\subsection{Description of the SDSS sample}

We studied the emission line data of galaxies selected from the SDSS DR6 database (AdelmanMcCarthy et al. 2008). The SDSS spectroscopic catalog contains galaxies of all types brighter than 17.77 mag in the $r$ band (Strauss et al. 2002) and a roughly volume-limited sample of luminous red galaxies with redshifts ranging up to $z \approx 0.45$ (Eisenstein et al. 2001). The spectra are taken using 3 arcsec diameter fibers, thus in the lowest redshift galaxies the spectroscopy only samples the central region. The data include redshifts, spectral type, measured characteristics of spectral lines etc. For a technical overview of SDSS see York et al. (2000).

The spectral line characteristics published in the spectroscopic catalog are evaluated by an automated pipeline. The pipeline is able to identify 48 spectral lines. In order to determine the fluxes the lines are fitted by Gaussian profiles. The database lists the fit parameters including position, height, width, EW and spectral continuum flux to each line. We use these values for our analysis.

The SDSS spectroscopic catalog also provides quantities that carry useful information on the spectral shape of galaxies in a compact form. Connolly et al. (1995) showed that the spectra of galaxies form a low-dimensional manifold. Using only a few parameters (say three) the spectra can be described with very good precision (99\% accuracy) and an objective spectral classification of galaxies is also possible. The spectroscopic pipeline applies this dimensional reduction technique as detailed in Connolly \& Szalay (1999) and Yip et al. (2004) to obtain the parameters, ecoeff $f_{i}$, $i=0 \ldots 4$ for each galaxy. They are the weights of the first five PCA eigenspectra of the SDSS galaxies. The derived quantity eclass $=\operatorname{atan}\left(-\operatorname{ecoeff}_{1} /\right.$ ecoeff $\left._{0}\right)$ characterizes the shape of the spectral continuum very well. Its increasing value corresponds to the rising blue end of the spectrum and decreasing 4000 A break, i.e. small/large eclass values indicate early/late types. (For illustration, see the left panel of Figure 11, which shows the correlation of eclass with the 
color $u-r$.

\subsection{Sample selection}

The sample for the present study was selected as follows. We selected objects that were classified by the SDSS photometric pipeline as galaxies. We restricted our investigations to the strongest 11 emission lines listed in Table 1. In order to include only reliable data we required the median $\mathrm{S} / \mathrm{N}$ in the $g^{*}$ band to be more than $10, \mathrm{sn}_{0}>10$ in the SpecObj data table of the SkyServer ${ }^{1}$ catalog science archive. For all galaxies included in our dataset we required that all lines listed in Table 1 are measured (125,832 objects). In order to exclude measurements with extremely large errors we set an upper cut in the errors of equivalent widths at $5 \mathrm{~A}$, this affected $5 \%$ of the parent sample. Our typical errors are between 0.2 and $0.4 \mathrm{~A}$, depending on line. The cleanness of data manifests e.g. in correct ratios of the known doublet lines. In some cases the line profile fit does not resolve the broad $\mathrm{H} \alpha \lambda 6565$ and its two close neighbors [N II] $\lambda 6550$ and [N II] $\lambda 6585$ correctly. In order to remove this systematic error, we excluded objects with blended $\mathrm{H} \alpha \lambda 6565$. An undesired effect of this sampling is the exclusion of galaxies that have some broad component around $\mathrm{H} \alpha$, e.g. Seyfert I galaxies. Our criterion to have all 11 lines reliably measured raises a concern about completeness in very low and very high metallicity ranges. In the former group [N II] lines might be unmeasurable, whereas in the latter group [O III] might be very weak or absent. We address this question later in Section 3.4. We also required sigma $<2$ for the [O II] $\lambda \lambda 3727,3730$, in order to exclude cases where the fit did not resolve the two lines but captured the blended doublet instead. We found these particular constraints the most suitable for obtaining both a clean and representative dataset.

Our final sample contains 40,312 galaxies. Since our selection criteria did not include any constraints on the sign of the emission line fluxes, our sample includes both emission line galaxies and objects with mainly absorption features. Some characteristics of the sample are shown in Figure 1, namely distribution of the data in redshift, absolute magnitude, color and eclass. Redshift ranges up to $z=0.3$, with an average value of $z=0.07$. In both color $u-r$ and continuum type eclass there are two groups of objects visible: red, early types at higher $u-r$ and low eclass and blue, late types at smaller $u-r$ and larger eclass. As described by Strateva et al. (2001) based on a study of SDSS galaxies, the two underlying groups of the bimodal distribution in color space can be separated by a single cut at $u-r=2.22$. According to this criterion, $41 \%$ of our galaxies are red and $59 \%$ are blue. The corresponding distribution of spectral types is manifested in eclass too. For more details on the distributions of galaxy spectral types in SDSS see Yip et al. (2004). We can distinguish between SF galaxies and active galactic nuclei (AGNs) using emission line diagnostics based on the line ratios $N 2=\log ([\mathrm{N}$ II $] \lambda 6585 / \mathrm{H} \alpha)$ and $O 3=\log ([\mathrm{O}$ III $] \lambda 5008 / \mathrm{H} \beta)$, which was introduced by Baldwin et al. (1981) (BPT). (For the distribution of our data on the BPT diagram

\footnotetext{
${ }^{1}$ http://skyserver.sdss.org
} 
see Figure 13, however, the explanation of the different symbols can be found later in Section 3.4.) In order to identify the two groups in our sample we adopt the AGN/SF separator of Kauffmann et al. (2003)

$$
O 3=0.61 /(N 2-0.05)+1.3 .
$$

Objects with $O 3$ values above this line are classified as AGNs, the rest are clessified as SFs. Based on this cut, nearly $50 \%$ of the sample are SF galaxies, $18 \%$ have an AGN-like emission pattern and over $32 \%$ of the objects cannot be classified as either because they have at least one of the four lines of Equation (1), in most cases $\mathrm{H} \beta$ having non positive EW's. These are objects with weak overall emission.

\section{Analysis of spectral lines}

\subsection{Equivalent width and spectral type distribution}

Figure 2 shows the distribution of the EW values of our sample plotted against the type parameter eclass (the smaller the redder). The data show the well-known tendency of emission lines becoming stronger from early to late types, see Figure 5 for a few examples. Star formation enhances both the blue color of late type galaxies and the strength of the emission lines. (This also makes it possible to use certain lines as measures of star formation, see e.g. Kennicutt (1998); Kewley et al. (2004).) This trend is obvious for all 11 analyzed lines. Early type galaxies located at negative eclass values show mainly absorption features and almost no emission independently of eclass. This group can be distinguished from late type galaxies situated mostly at positive eclass values, whose emission tends to rise with eclass. However, this tendency is not the same for the different lines, which originates in different physics of formation. We also see galaxies that have seemingly strong absorption at $\mathrm{H} \gamma \lambda 4342$ but not at $\mathrm{H} \alpha \lambda 6565$, which is puzzling as typically one expects the absorption EW at $\mathrm{H} \alpha \lambda 6565$ to be about $60 \%$ of that at $\mathrm{H} \gamma \lambda 4342$. Since our EW's are the sum of emission and absorption, this probably means that the $\mathrm{H} \alpha \lambda 6565$ absorption is filled up by the emission of these galaxies. This effect is enhanced by measurement uncertainties as well. Seeming strong absorption values at $[\mathrm{O}$ III $]$ and $[\mathrm{S}$ II $]$ are also present due to measurement errors.

\subsection{Orthogonal approach}

We try to quantify the common trends and differences in the variation of the nebular emission pattern using PCA of the EW data. They characterize the emission strength physically as they do not depend on distance and the effect of the galactic reddening is canceled by normalization. At the same time, EW's are affected by the intrinsic reddening caused by an inhomogeneous distribution of star, gas and dust components (Calzetti et al. 1994; Charlot \& Fall 2000). Because we are interested in data as they are observed in photometric measurements, we choose not to correct for the intrinsic reddening. 
PCA is a linear transformation of data vectors to the eigensystem of their correlation matrix. It results in an uncorrelated representation of the data, and makes it possible to identify the most relevant directions of variation by ranking them according to their information content.

PCA of our data was carried out as follows. We represented the EW's of each galaxy with an $M=11$ dimensional vector $\mathbf{y}$, with the average $\overline{\mathbf{y}}$ subtracted. Diagonalizing the covariance matrix we obtained the orthonormal set of $M$ eigenvectors or principal components (PCs) $\mathbf{e}^{k}$. We ordered them by their eigenvalues $\lambda^{k}$ (normalized to unit trace) since they express the relative information content of each eigenvector. For each galaxy the expansion coefficients of the vector $\mathbf{y}$ form the new $M$ dimensional vector $\mathbf{c}$ :

$$
\mathbf{y}=\sum_{k=1}^{M} c_{k} \mathbf{e}^{k} .
$$

The transformation of vector $\mathbf{y}$ to vector $\mathbf{c}$ corresponds to a simple rotation of the data vectors to the basis where their correlation matrix is diagonal. Inverting the transformation we obtain the original vectors again. However, if we truncate the expansion coefficients at some $m<M$, the data will not be exactly restored. The effect of omitting the $k$ th principal component is the reduction of the variance of the truncated EW estimator

$$
\mathbf{y}^{(m)}=\sum_{k=1}^{m} c_{k} \mathbf{e}^{k},
$$

by $\lambda^{k}$ with respect to the original distribution of the data. Hence, the eigenvalues are actually a measure of the importance of each eigenvector to reconstructing the real distribution of the data.

\subsection{The principal components}

Figure 4 shows the results of PCA of the EW vectors. The average EWs for each of the 11 lines (vector $\overline{\mathbf{y}}$ ) are plotted in the top panel. Below these are the first five eigenvectors $\mathbf{e}^{k}$ ordered by their eigenvalues. Their information content is $89.1 \%, 7.8 \%, 1.8 \%, 0.7 \%$ and $0.2 \%$ of the total variance, respectively. The numerical results are summarized in Table 2 . The meaning of each PC can be easily interpreted by comparing the weight of the $i$ th line in the $k$ th eigenvector $e_{i}^{k}$ with the mean EW of the $i$ th line $\bar{y}_{i}$.

The first eigenvector $\mathbf{e}^{1}$ is very similar to the average vector $\overline{\mathbf{y}}$. It means, the most important variation in emission line EWs is simply a constant multiplicative factor in the amplitude that varies from galaxy to galaxy. A larger $c_{1}$ indicates stronger nebular emission. The PC is dominated by the strongest line $\mathrm{H} \alpha \lambda 6565$.

The second eigenvector $\mathbf{e}^{2}$ represents mostly the [O III] $\lambda \lambda 4960,5008$ and nitrogen lines. The coefficients of [O III] $\lambda \lambda 4960,5008$ and [N II] $\lambda \lambda 6550,6585$ have opposite signs. The same holds for [O II] $\lambda \lambda 3727,3730$ and [N II] $\lambda \lambda 6550,6585$ in the eigenvector $\mathbf{e}^{3}$. This enables the nitrogen emission lines to vary independently of oxygen in the reconstructed emission-line spectrum. The EW data 
(Figure 2) show that [N II] emission grows continuously, slowly from early to late types, while the oxygen lines have a stronger type dependence becoming steep especially for the extremely blue galaxies. Due to the higher ionization degree, the behavior of the [O III] doublet as a function of type is different from the other lines: while showing no significant emission at low and moderate eclass values, there is a steep rise at eclass $>0.5$. Nitrogen in these components is relatively strong compared to the Balmer lines, thus both $e_{2}$ and $e_{3}$ influence the $[\mathrm{N} \mathrm{II}] / \mathrm{H} \alpha \lambda 6565$ ratio.

These eigenvectors do not significantly change the ratios of lines in the same doublet since their weights normalized by their average values are nearly equal. The constant ratios have physical reasons and it is a strong effect which persists in these components.

In $\mathbf{e}^{4}$ the two lines of the [O II] $\lambda \lambda 3727,3730$ doublet are represented with opposite signs. The effect of this component is to change their ratio. This eigenvector reflects the measurement errors of the $[\mathrm{O}$ II $] \lambda \lambda 3727,3730$, which is difficult to deblend with the resolution of SDSS spectroscopy.

Vector $\mathbf{e}^{5}$ contains mostly [N II] $\lambda \lambda 6550,6585$, with some weak Balmer and oxygen lines. Thus we expect that it might influence the precise reconstruction of [N II] lines.

Doublet lines in some higher PCs often appear with opposite sign, which is mainly the effect of errors. We do not detail the further components as their variance is less than $0.2 \%$ for each, they are dominated by noise.

\subsection{Eigenspace representation of emission line data}

The distribution of the data in the subspace of the first three PCs is shown in Figure 5. The range occupied by galaxies is approximately a two-dimensional curved manifold. Most of the objects form a triangular region that is closely parallel to the axes $\mathbf{e}^{1}$ and $\mathbf{e}^{2}$ and closely perpendicular to $\mathbf{e}^{3}$, having small $c_{3}$ coordinates. The distribution also has a 'head' at the lower end in $c_{1}$ and a 'tail' having large $c_{1}$ values. The galaxies in the 'tail' also have significant contribution from the third $\mathrm{PC}$ but are still located on the curved surface which is a continuation of the main locus described above. The fact that PCA overestimates the dimensionality of the data is a known limitation of the method. It is because PCA is a linear transformation while the physics of emission lines generates nonlinear structure (see Figure 2).

As shown in Figure 6, the points in the triangular main locus embedded in the subspace of the first three eigenvectors can be generated by two vectors originated in a point $C$ (Chan et al. 2003). Their coordinates in $\left(c_{1}, c_{2}, c_{3}\right)$ space are: $C=(-22,5,0), \mathbf{u}=(102,-45,15)$, $\mathbf{v}=(102,15,-15)$. The origin and the vectors correspond to certain emission pattern recovered from three PCs according to Equation (3). As the figure shows, the origin has almost no emission. Thus, since the region is situated approximately in the $\left(\mathbf{e}^{1}, \mathbf{e}^{2}\right)$ plane, the EW values in these two vectors alone can give us some idea of physical interpretation of the first two PCs.

As indicated in Section 3.3, the overall strength of the emission is manifested in the first 
coefficient $c_{1}$. We define the relative emission line flux fraction $\mu$ as the total emission line flux normalized by the continuum flux within the range $3728-6733 \mathrm{~A}$. If we plot this quantity for each galaxy in the plane $\left(\mathbf{e}^{1}, \mathbf{e}^{2}\right)$, we can see the monotonic growth of $\mu$ with $c_{1}$ (Figure 7 ). It is apparent that the gradient of $\mu$ is almost parallel to the axis $\mathbf{e}^{1}$. The inset plot shows a linear relation between $\mu$ and $c_{1}$; the 'head' of the distribution has an emission flux fraction of less than $1 \%$, while the largest $c_{1}$ galaxies have $\mu \approx 0.5$, i.e., equal flux contribution from continuum and emission lines in the analyzed wavelength interval.

Another striking effect in Figure 6 is that while oxygen lines are relatively strong, the nitrogen emission is weak in vector $\mathbf{v}, \mathbf{v}-\mathbf{u}$, the difference, shows negative [N II] lines. This indicates a difference in metallicity. We estimated the ratio of oxygen and hydrogen abundances from the ratio of [N II] $\lambda 6585$ and $\mathrm{H} \alpha$ fluxes using the empirical formula of Pettini \& Pagel (2004).

$$
12+\log (\mathrm{O} / \mathrm{H})=8.9+0.57 \log ([\mathrm{N} \text { II }] \lambda 6585 / \mathrm{H} \alpha) \text {. }
$$

As this estimator is calibrated for H II galaxies we excluded objects dominated by non-thermal emission from this analysis by requiring $\log ([\mathrm{N}$ II $] \lambda 6585 / \mathrm{H} \alpha)<-0.3$. Figure 8 shows that the vector $\mathbf{v}-\mathbf{u}$ points approximately in the direction of the negative metallicity gradient: at a fixed contribution from vector $\mathbf{u}$ the objects tend to have smaller metallicities if the mixing ratio of $\mathbf{v}$ is larger, i.e. upwards in the diagram. The 'head' has large metallicity values which indicate old galaxies.

In Figure 6 data are plotted only up to $c_{1}=300$, however, the 'tail' of the distribution reaches nearly $c_{1}=700$. The vector $\mathbf{w}$ together with the previous two vectors generates the long tail. Its endpoint $E[\mathrm{~A}]=(500,250,80)$ is out of the range of the figure, so only a fifth of the vector, w/5 is plotted. Point $E$ represents galaxies with extremely strong nebular emission. Since vector w carries very strong EW values compared to vector $\mathbf{v}$ (or $\mathbf{u}$ ), the spectrum of the point $E$, as well as the points near the large $c_{1}$ end of the distribution, is very similar to that of vector $\mathbf{w}$. Very strong Balmer lines and [O III] $\lambda \lambda 4960,5008$, weak [O II] $\lambda \lambda 3727,3730$, and nitrogen deficiency can be observed. Even though our data are not corrected for reddening, the large [O III]/[O II ] ratio is a real, strong effect. It implies a large ionization parameter of the emitting gas, which increases with $c_{1}$ at large $c_{1}$ values. In summary, the galaxies of the 'tail' have extremely strong emission, very low metallicities and high ionization parameters which indicates that they are young bursting objects.

Figure 9 shows the color $u-r$ in the subspace of the first and the second PC. A red 'head' and a blue 'tail' can be seen, and the color becomes continuously bluer toward large $c_{1}$ values. This indicates a close relation of the distribution in the emission line PC space to the bimodality seen in color. The histograms at the bottom of the diagram indicate the distribution of red and blue galaxies defined by the $u-r=2.22$ cut of Strateva et al. (2001). The transition between the two distributions is around $c_{1} \approx-15$ where we have an equal number of red and blue galaxies in our sample. By a cut at $c_{1}=-15$ we can select $93 \% / 86 \%$ of blue/red galaxies, with around $10 \% / 11 \%$ contamination from the other group, respectively. 
The color $-c_{1}, c_{2}$ correlation indicates that $c_{1}$ must be strongly correlated to the continuum shape of the spectral energy distribution (SED). Figure 10 shows the relation between eclass and the first principal components. The $c_{1}$-eclass relation is very similar to the relation of $c_{1}$ and $-(u-r)$. This is not surprising as they both measure the same effect: the difference between the intensity of the blue and the red end of the spectrum. Their relation is illustrated in Figure 11 and resembles two linear relations, one for the early type objects and the other for the late type objects. The $u-r=2.22$ cut in color corresponds to a cut at eclass $\approx-0.05$. As shown by the histogram in Figure 11 (bottom), it also roughly corresponds to the inflection point of the eclass distribution. The color distribution is also shown projected to the right margin of the diagram. In fact, the separator lines lie somewhat blueward from the inflection points in both eclass and color, which might be the effect of undersampling of early types by our selection. If we consider eclass $=-0.05$ as the separator of early and late spectral types, we can see that the separation is even slightly clearer than in the case of colors. The cut selects $93 \% / 88 \%$ of late/early spectral types with a fraction of $8 \% / 11 \%$ of early/late type objects misclassified by the cut. The correlation of eclass and the relative emission parameter $\mu$ is shown in the right panel of Figure 11. We will discuss the issue of the emission line PC's and spectral type in more detail later in Section 4.

The absolute magnitude in the $r$-band is shown in Figure 12. Apart from the large scatter, the objects are generally fainter at larger $c_{1}$. At the largest $c_{1}$ values only low luminosity objects are present, with typical $M_{r} \approx-17$. This is in concordance with the earlier studies of SDSS galaxies by Tremonti et al. (2004) who found that the most metal deficient galaxies are faint.

Figure 13 shows the connection of the first two PCs to the AGN/SF diagnostic diagram. The line ratios $N 2$ versus $O 3$ are plotted in the left panel, together with the AGN/SF separator line of Equation (1). The points are colored by the first principal component, using a cut at $c_{1}=-5$ which appears to be the most reliable c-cut separating the AGN from the SF. Red symbols denote $c_{1}<-5$, blue symbols indicate $c_{1}>-5$. This separator lies blueward of the color or spectral type separator, as a significant fraction of AGNs has a bluer/later spectral type and has more contribution from emission lines than do the average red galaxies. Up to the mixing at the lower edge of the BPT diagram, the two $c_{1}$ regions roughly agree with the AGN/SF separation: the $c_{1}$ cut selects $82 \% / 84 \%$ of SFs/AGNs with $6.5 \% / 37 \%$ contamination from the other group, respectively. We note that the $c_{1}=-5$ cut selects $92 \% / 58 \%$ of SF/AGN as defined by Stasinska et al. (2006), with 34\%/10\% contamination from the other group, respectively. The cut of Stasinska et al. (2006) classifies more objects as AGN and is therefore more consistent with a higher cut, about $c_{1} \approx 0$. The separation of the types is not well defined partly because of the mixing of SF and AGN activity in some low emission galaxies. In the right panel of Figure 13 AGN (red) and SF galaxies (blue), selected by Equation (1) are plotted on the $c_{1}: c_{2}$ diagram. The vertical line is at $c_{1}=-5$. The plot shows the same subset of objects as in the left panel, which means all galaxies where Equation (1) is not applicable are excluded. They are low emission objects having non-positive $\mathrm{H} \alpha, \mathrm{H} \beta$, [N II] $\lambda 6585$ or [O III $] \lambda 5008$. Nearly all of them $(99.9 \%)$ are at $c_{1}<-5$. Note that low nebular emission galaxies are underrepresented due to the selection criteria. If present, the missing galaxies would 
populate the 'head' too. We also note that AGN with broad $\mathrm{H} \alpha$ emission lines are excluded from our sample. With our present sample, the 'head' (at $c_{1}<-5$ ) consists of low emission objects, $31 \%$ of which could be classified as AGNs. The plot confirms that the 'tail' consists of SF galaxies. The classification using $c_{1}$ and $c_{2}$ is also reminiscent of the $(\mathrm{W}(\mathrm{H} \alpha),[\mathrm{N} \mathrm{II}] / \mathrm{H} \alpha)$ diagram proposed by Cid Fernandes et al. (2010), which distinguishes among SFs, AGNs and LINER-like galaxies, since $c_{1}$ is closely connected to the $\mathrm{H} \alpha \mathrm{EW}$. However, because of our selection criteria on equivalent width, we have very few LINER-like galaxies in our sample, these are situated at the lowest $c_{1}$ values.

Now we return to the question of missing low and high metallicity objects, rejected because of the EW criterion. According to a check carried out in both a low $(7.5-7.6)$ and a high $(8.6-$ 8.8) metallicity bin, indeed, both ranges are underrepresented by about one-third in our selection, if compared to the rejected group. However, there is still a sufficient number of both low and high metallicity galaxies in the selected sample for correlation analyses. Although for the relative occurence of the emission patterns our sample might not be conclusive, it is still suitable for studying the relations between emission lines and other observables.

We can conclude that PCA isolates two groups of objects: red, early spectral type, low emission, high metallicity, bright galaxies, with a significant fraction of AGNs in the 'head' and the rest of the objects which are blue, late spectral type, high emission, lower metallicity, fainter, star forming galaxies. In the main locus, there is a gradient of all quantities listed above. All these characteristics get continuously more prominent and reach extreme values toward the end of the 'tail'.

The low effective dimensionality of the emission pattern found by PCA is not surprising. As already discussed in the Introduction, the reason is the dominance of only a couple of mechanisms. The largest impact on the emission line flux pattern has the relative importance of SF vs. AGN, the second most important influencing factor is metallicity. These generate the effectively twodimensional locus of galaxies in the emission line space.

\subsection{Reconstructing spectral lines}

We studied the effect of truncation of the principal component basis on the restored EWs. We examined the convergence of the truncated EW estimator (Equation(3)) as a function of the number of eigenvectors used for the reconstruction. The error of the estimation can be characterized using the residuals added in quadrature over all lines

$$
\left(\Delta y^{(m)}\right)^{2}=\sum_{i=1}^{11}\left(y_{i}^{(m)}-y_{i}\right)^{2} .
$$

Figure 14 shows $\Delta y^{(m)}$ averaged over the whole sample as well as for three $c_{1}$ bins. Remember that larger $c_{1}$ values mean stronger emission and later spectral type. For the earliest bin $c_{1}<0$ the contribution of the emission lines is so small that estimating by average $(m=0$ case) produces 
larger error than ignoring the emission lines, i.e. setting $\mathrm{EW}=0$ for all lines. We demonstrate the error of the estimation by zero EWs for eclass $<-0.05$ galaxies by an arrow at the left margin of the diagram. For early spectral types, ignoring the emission would mean a $10 \mathrm{~A}$ error if summed over all lines.

If we drop all eigenvectors with eigenvalues less than $1 \%$, we will have the first three PC's. Their total percent variance for the case $m=3$ is $98.6 \%$. Using the first three components we can reconstruct the total emission with $3 \mathrm{~A}$ average precision. The error is type dependent, its absolute value increases with $c_{1}$. For the strongest emission bin the average $\Delta y^{(3)}$ is $8 \mathrm{~A}$. However, unlike the absolute error, the relative error defined as

$$
\delta y^{(m)}=\Delta y^{(m)} /|y|
$$

is smaller for stronger emission objects. The average relative error is $25 \%$ for the whole sample, dominated by the error of objects having small EW values. For the extremely strong emission bin it is only $5 \%$.

We show the errors of the strongest, most important lines individually. In Figure 15 we showed the convergence of the residuals of the individual EWs of [O II] $\lambda 3730,[\mathrm{O}$ III] $\lambda 5008,[\mathrm{~N}$ II] 26585 and $\mathrm{H} \alpha \lambda 6565$ :

$$
\Delta y_{i}^{(m)}=\left|y_{i}^{(m)}-y_{i}\right|
$$

averaged over the sample. Similar to the overall emission represented by the summed residuals, all the individual lines are reconstructed with an average error not larger than $2 \mathrm{~A}$ using three PCs (or not larger than $4 \mathrm{~A}$ using two PCs). For the early type objects at eclass $<-0.05$, we repeated the estimation by zero emission line flux similar to Figure 14. If we set all EWs to zero, the errors of the individual lines are still below 5Afor this group, as shown by the errors in the left margin of the plot.

We checked the effect of the truncation on physical quantities such as metallicity and emission flux fraction if these were determined using three-PC-reconstructed EW data. The relative emission line flux fraction $\mu$ is plotted in Figure 16, truncated to the first three PCs versus the original. The rms error of the reconstruction is as small as 0.001 which means an estimation of the emission line flux fraction within a precision of $0.1 \%$. The lines reconstructed from the first three PC's obey the known ratios of the doublet lines, especially [O III] $\lambda \lambda 4960,5008$ and [N II] $\lambda \lambda 6550,6585$ with a relatively good precision. The line ratio $[\mathrm{N}$ II $] \lambda 6585 /[\mathrm{N}$ II $] \lambda 6550$ in the reconstruction using the first three eigenvectors is 3.26 , whereas the fitted ratio in the original data is 3.23 . For [O III], the fitted [O III] $\lambda 5008 /[\mathrm{O}$ III] $\lambda 4960$ ratio is 3.06 for the reconstructed data and 3.01 for the original data. These features are so strong that only higher PC's begin to violate them by including noise components. However, the flux ratios of lines that are not in the same doublet are not restored precisely. If we want to use the [N II] $\lambda 6585 / \mathrm{H} \alpha$ ratio for diagnostic purposes to distinguish between thermal emission of $\mathrm{HII}$ regions from the AGN-like emission, we need the first three PCs and the fifth PC as well. The fifth eigenvector is the one that makes possible an efficient fine-tuning of this ratio as it contains $\mathrm{H} \alpha$ and [N II] with opposite signs. Figure 17 shows that metallicity estimation 
with the first three PC's has a relatively large error, which can be suppressed by the inclusion of the fifth eigenvector. The rms errors of the reconstructed metallicity are 0.23 and 0.13 without and with $\mathbf{e}_{5}$, respectively. The reconstruction is less precise at high metallicities. This is because of the weak $\mathrm{H} \alpha$ and $\mathrm{H} \beta$ lines, since, as described in Section 3.4, these are typically early type galaxies.

\section{Correlation of spectral lines and continuum features}

The motivation of this study was to explore the connection between the continuum spectral type and the emission pattern of the emission line galaxies. Although it is clear that there is no one-by-one relation, as a first step we disregard the variations and focus on the systematic trends. For the practical applications, we would like to make predictions about theemission lines based on continuum parameters. This can then be used, e.g., to add emission lines to galaxy model SED's which only contain stellar populations.

We characterize the continuum spectrum with the three most informative coefficients of the spectral principal component expansion ecoef $f_{0}$, ecoeff $f_{1}$ and ecoef $f_{2}$. We investigate their link to the first three emission line PCA coefficients $c_{1}, c_{2}$ and $c_{3}$ which proved to be essential in reconstructing the emission with sufficient accuracy. Figure 18 illustrates how these parameters are linked to each other. The data points in all diagrams are colored by ecoeff $f_{0}$, ecoeff $f_{1}$ and ecoef $f_{2}$. For late type objects with significant emission lines a mapping from the first three ecoeff's to the first three $c_{i}$ appears to be possible.

The correlation of each of the first three $c_{i}$ are plotted against eclass in the top panels of Figure 19. The first coefficient $c_{1}$ exhibits the strongest correlation with the continuum features. This coefficient also has the largest information content. There are apparent systematic trends in $c_{2}$ and $c_{3}$ as well. Given a continuum spectral type, we can determine the expectation values and variances of the emission line EW's based on these empirical relations. The ontinuum PCs ecoeff $f_{0}$-ecoeff $f_{2}$ carry even more information that can be used to establish an empirical relation. As indicated in the previous section and as one can see in the top panels of Figure 19, early type galaxies might be fitted by constant values which would yield nearly zero flux. However, now we choose to treat all data equally. We have checked that the two approaches do not make a significant difference. We fit a second order polynomial of three variables ecoeff $f_{0}$, ecoef $f_{1}$ and ecoef $f_{2}$ to each of $c_{1}, c_{2}$ and $c_{3}$,

$$
c_{i}=\alpha_{i}+\sum_{k=0}^{2} \beta_{i}^{k} \operatorname{ecoeff}_{k}+\sum_{k=0}^{2} \sum_{l=k}^{2} \gamma_{i}^{k l} \operatorname{ecoeff}_{k} \operatorname{ecoeff}_{l} .
$$

The fitted coefficients are listed in Table 3. We can use this empirical relation to estimate emission properties solely from continuum features of the spectra. The residuals of the fit, $c_{i}($ fit $)-c_{i}$, which characterize the goodness of the estimation, are shown on the bottom panels of Figure 19. The rms error of the fit residuals is plotted as a function of spectral type too. The origin of the scatter is mainly the cosmic variance, which includes the effect of geometry (Yip et al. (2010)) and other 
physical parameters not fully covered by the spectral classification parameter. The scatter becomes large toward the largest eclass values. However, as the flux values themselves are large here, the resulting relative flux error is smaller than for earlier types.

For practical applications, we also list the ecoeff $f_{i}$ fits for four lines (H $\alpha$, [O II], [O III] and $[\mathrm{N}$ II] $)$ in Table 4. For each doublet, we added up the values of the two individual lines. However, this approach does not recover flux information in such a compact form as fitting the PCs. While with Table 3 we restore $99.7 \%$ of the emission line flux with just three components, Table 4 gives only $94.4 \%$ of the flux with four fits to a total of seven lines, and does not contain information on individual lines except for $\mathrm{H} \alpha$.

We use the fitted $\tilde{c_{i}}$ values of Table 3 to reconstruct the emission lines analogously to Equation (3):

$$
\tilde{\mathbf{y}}=\sum_{k=1}^{3} \tilde{c}_{k} \mathbf{e}^{k},
$$

We compare the emission lines coming from this estimator with the measured values and calculate the errors described in Equations (5) - (7), by substituting $\tilde{y}$ for $y^{(m)}$. The errors of this prediction are plotted in Figures 14 and 15 with small arrows at the right margin of each plot. We find that the strength of the total nebular emission can be predicted from the spectral continuum with an average accuracy of $5 \mathrm{~A}$ or $40 \%$ for the entire sample. However, for the objects of greatest interest those having significant emission - the relative precision is better. The average errors are $10 \mathrm{~A}(20 \%)$ for the $0<c_{1}<100 \mathrm{bin}$, and $25 \mathrm{~A}(\approx 10 \%)$ for the strongest emission bin $\left(c_{1}>100\right)$. Estimating by average only, without using the continuum dependence the error can be as large as $\approx 100 \%$.

We can estimate, how well the reconstruction of emission lines works in terms of photometry. We simulate photometry by convolving the SDSS filters with the spectra of the objects. We investigate the impact of emission lines by omitting them, convolving just the continuum and comparing these magnitudes with the values obtained from the entire spectrum (continuum+lines). The results for the $g, r$, and $i$ bands are shown in the first and second rows of Figure 20. The impact of the nebular lines is strongly type-dependent. For the strongest emission objects at high eclass, the magnitude difference due to the lack of emission lines can reach $0.5^{m}$ in the $g$ band. This is the effect of [O III] $\lambda \lambda 4960,5008$ and [O II] $\lambda \lambda 3727,3730$. The largest difference in the $r$ and $i$ bands is $\approx 0.2^{m}$. The significance of the emission line contribution becomes striking when compared to the typical photometric uncertainties. (For an $r \approx 19^{m}$ galaxy the photometric data have a typical error of $0.03^{m}, 0.025^{m}$ and $0.07^{m}$ in the $g$, rand $i$ bands, respectively.) Note the redshift dependence due to lines being redshifted into and out of the filters. Most apparent is the $r$ band, the low redshift hump comes mainly from $\mathrm{H} \alpha$ and the high redshift hump from [O III] $\lambda \lambda 4960,5008$, which are then redshifted into the $i$ band. On the bottom three plots we estimated the emission lines from ecoef $f_{i}$, using the fitted $\tilde{c}_{i}$ values and Equation (9). We carried out simulated photometry with continuum + predicted lines, and compared this with photometry simulated with real emission lines. The results show that the prediction approximates the original values with a maximum error $\approx 0.1^{m}$ in $g$ for the strongest emission objects and $0.05^{m}$ for $r$ and $i$. The rms error is of the order of 
$0.01^{m}$ for the extremely high emission bin eclass $>0.6$ and $\approx 0.001^{m}$ for eclass $<0.6$. (We note that the early types at eclass $<-0.05$ have errors not larger than this even if no emission line flux is added.) This precision is sufficient for the most photometric applications. For example, one can add spectral lines to model SEDs of stellar populations or any empirical spectra with missing emission lines in a way that makes the continuum features and the emission pattern consistent with the observations.

As an example of practical applications a similar method has been successfully applied to improve the spectral templates used in SDSS photometric redshift estimation. In SDSS, we used a hybrid photometric redshift (photo-z) algorithm that holds the advantages of both the template fitting and empirical approaches. Here we use semi-empirical spectral templates that are based on the Coleman, Wu, \& Weedman (1980) (CWW) empirical spectra with an additional extension of the UV and IR ends with Bruzual-Charlot model spectra. These are, actually, the most widely used templates in the empirical template fitting photo-z applications. In our approach, the templates are iteratively trained on a reference set of galaxies with known redshifts and photometry, so that they match better the photometry of the SDSS data (Budavári (2000)). The quality of photo-z

estimation clearly improves by the use of repaired templates. However, we see instabilities during the iterative procedure around the positions where our paper's analysis would predict a strong line. We attribute this effect to the discrepancy between the continuum-line correlations of the CWW templates and the SDSS data sample. To correct for this, we used a modified algorithm, where the emission lines in the CWW spectra are replaced using the empirical continuum-line correlations, discussed in this study. This modified method was indeed used in SDSS data releases DR4, DR5 and DR6 (Adelman-McCarthy et al. (2006, 2007, 2008)), where this ingredient has reduced the error on the photo-z estimate by $10 \%$ for the bluest galaxies. In Table 5 , we show the effect of this procedure on the photo-z results in detail. The photo-z type $t$ parameterizes the spectral type, with 0 denoting the reddest galaxies, and 55 denoting the bluest galaxies. We list the rms photo-z error as a function of type, for the cases when the procedure is carried out using the original or the modified CWW spectra. While the estimate of the early type objects remains unchanged as expected, there is an improvement increasing with type. Beyond this, we consider the stabilization of the training procedure as the main achievement.

We have shown how the relations shown in Figure 19 can be used to predict the expectation values of EWs based on the spectral type. The variance may serve as additional information when a simulated distribution of the emission pattern is generated.

\section{Conclusions}

We considered a sample of over 40,000 SDSS galaxy spectra (emission lines and continua) coming mostly from cores due to the aperture effect of the survey. Using PCA of EWs of the 11 selected emission lines, we found that nearly $99 \%$ of information is included in the subspace generated by the first three eigenvectors. They reconstruct emission line fluxes within a precision 
of $5 \%-25 \%$, depending on spectral type. We found that based on a three-dimensional eigenspace representation of continuum spectra there is a simple way of estimating the most probable emission line pattern and its variation, which makes it possible to determine the total photometry from the continuum spectrum in the investigated bands with a precision $<0.1^{m}$. The applications include the comparison of photometric observations with models, e.g., determining K-corrections and absolute magnitudes. The prescription for adding lines on template spectra has been successfully applied to improve the precision of photometric redshift estimation.

The authors acknowledge support from the following grants: OTKA-MB08A-80177, MRTNCT-2004-503929, NKTH: RET14/2005, KCKHA005, and Polányi.

Funding for the creation and distribution of the SDSS Archive has been provided by the Alfred P. Sloan Foundation, the Participating Institutions, the National Aeronautics and Space Administration, the National Science Foundation, the U.S. Department of Energy, the Japanese Monbukagakusho, and the Max Planck Society. The SDSS Web site is http://www.sdss.org/. The SDSS is managed by the Astrophysical Research Consortium (ARC) for the Participating Institutions. The Participating Institutions are The University of Chicago, Fermilab, the Institute for Advanced Study, the Japan Participation Group, The Johns Hopkins University, Los Alamos National Laboratory, the Max-Planck-Institute for Astronomy (MPIA), the Max-Planck-Institute for Astrophysics (MPA), New Mexico State University, Princeton University, the United States Naval Observatory, and the University of Washington. 


\section{REFERENCES}

Adelman-McCarthy, J.K. et al.2006, ApJS 162, 38

Adelman-McCarthy, J.K. et al.2007, ApJS 172, 634

Adelman-McCarthy, J.K. et al.2008, ApJS 175, 297

Baldwin, J.A, Phillips, M.M., \& Terlevich, R. 1981, PASP, 93, 5

Bolzonella, M., Miralles, J.-M., \& Pelló, R. 2000, A\&A 363, a7,-492

Brammer, G.B., van Dokkum, P.G., \& Coppi, P. 2008, ApJ, 686, 1503

Brinchmann, J., Charlot, S., White, S. D. M., Tremonti, C., Kauffmann, G., Heckman, T., Brinkmann, J. 2004, MNRAS, 351, 1151

Bruzual, G., \& Charlot, S. 2003, MNRAS, 344, 1000

Budavári, T., Szalay, A. S., Connolly, A. J., Csabai, I., Dickinson, M. 2000, AJ, 120, 1588

Budavári, T.et al.2001, AJ, 122, 1163

Budavári, T. 2009, ApJ, 695, 747B

Calzetti, D., Kinney, A.L., \& Storchi-Bergmann, T. 1994, ApJ, 429, 582

Chan, B.H.P., Mitchell, D.A., \& Cram L.E. 2003, MNRAS, 338, 790

Charlot, S. \& Fall, S.M. 2000, ApJ, 539, 718

Charlot, S. \& Longhetti, M. 2001, MNRAS, 323, 887

Coleman, G. D., Wu, C.-C., \& Weedman, D. W. 1980, ApJS, 43, 393

Connolly, A.J, Szalay, A.S., Bershady, M.A., Kinney, A.L., \& Calzetti, D. 1995, AJ, 110, 1071

Connolly, A. J. \& Szalay, A. S. 1999, AJ, 117, 2052

Csabai, I. et al.2003, AJ, 125, 580

Eisenstein, D. et al. 2001, AJ, 122, 2267-2280

Ferland, G. J. 1996, Hazy, a Brief Introduction to CLOUDY (Lexington, KY: Univ. of Kentucky Internal Report), 565

Cid Fernandes, R., Stasinska, G., Schlickmann, M. S., Mateus, A., Vale Asari, N., Schoenell, W., Sodré, L. 2010, MNRAS, 403, 1036

Fioc, M., \& Rocca-Volmerange, B. 1997, A\&A, 326, 950 
Firth A.E., Lahav O., \& Somerville R.S. 2003, MNRAS, 339, 1195

Kaiser, N. and PanSTARRS Team, 2005, BAAS, 37, 1409

Kauffmann, G. et al.2003, MNRAS, 346, 1055

Kennicutt, R. C., Jr. 1998, ARA\&A, 36, 189

Kewley, L. J., Geller, M. J., \& Jansen, R. A. 2004, AJ, 127, 2002

Moy, E., Rocca-Volmerange, B., \& Fioc, M. 2001, A\&A, 365, 347

Panuzzo, P., Bressan, A., Granato, G. L., Silva, L., \& Danese, L. 2003, A\&A, 409, 99

Pettini, M. \& Pagel, B.E.J. 2004, MNRAS, 348, 59

Sodré, L. \& Stasinska, G. 1999, A\& A, 345, 391

Stasinska, G. \& Leitherer, C. 1996, ApJS, 107, 66

Stasinska, G. 1990, A\&AS, 83, 501

Stasinska, G. \& Sodré, L. 2001, A\& A, 374, 919

Stasinska, G., Cid Fernandes, R., Mateus, A., Sodré, L., Asari, N. V. 2006, S, 317, 972

Strateva, I. et al. 2001, AJ, 122, 1861

Strauss, M. et al. 2002, AJ, 124, 1810-1824

Tremonti, C. et al. 2004,ApJ, 613, 898

Tyson, J. A. 2002, Proc. SPIE, 4836, 10

Wolf C., et al.2004, A\&A, 421, 913

Yip, Ching-Wa et al. 2004, AJ, 128, 585

Yip, C.-W., Szalay, A. S., Wyse, R. F. G., Dobos, L., Budavári, T., i, I. 2010, ApJ, 709, 780

York, D. et al. 2000, AJ, 120, 1579 

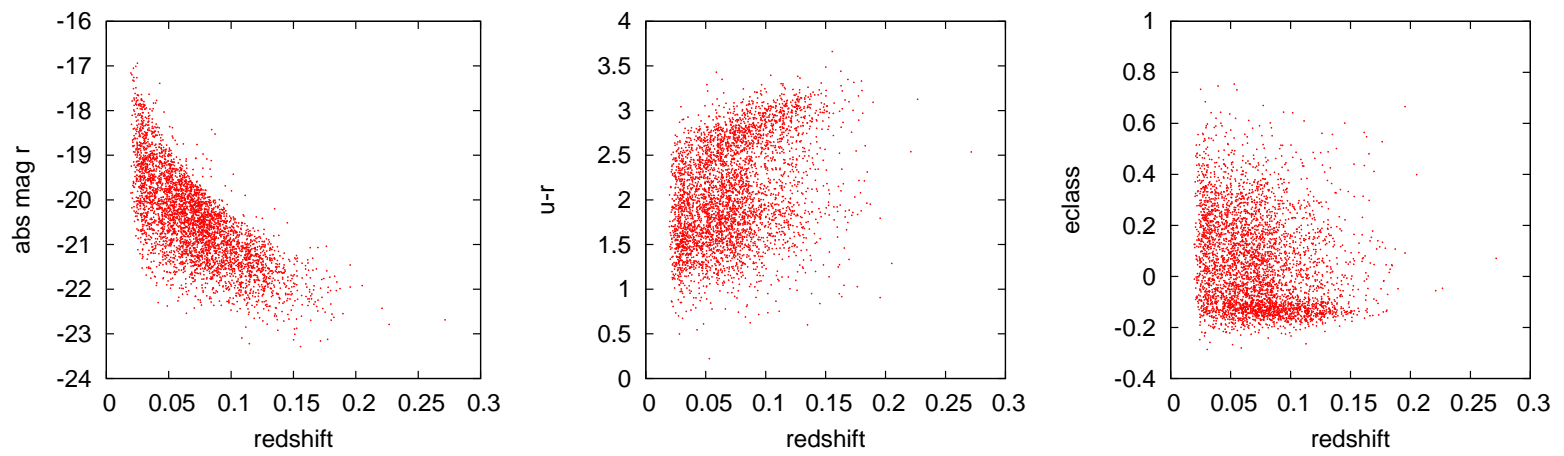

Fig. 1.- Distribution of absolute magnitude (left), color $u-r$ (middle) and the continuum spectral type parameter eclass (right) vs. redshift shown for a $10 \%$ random subset of our sample. Two distinct groups of galaxies can be identified in both color and spectral type. 

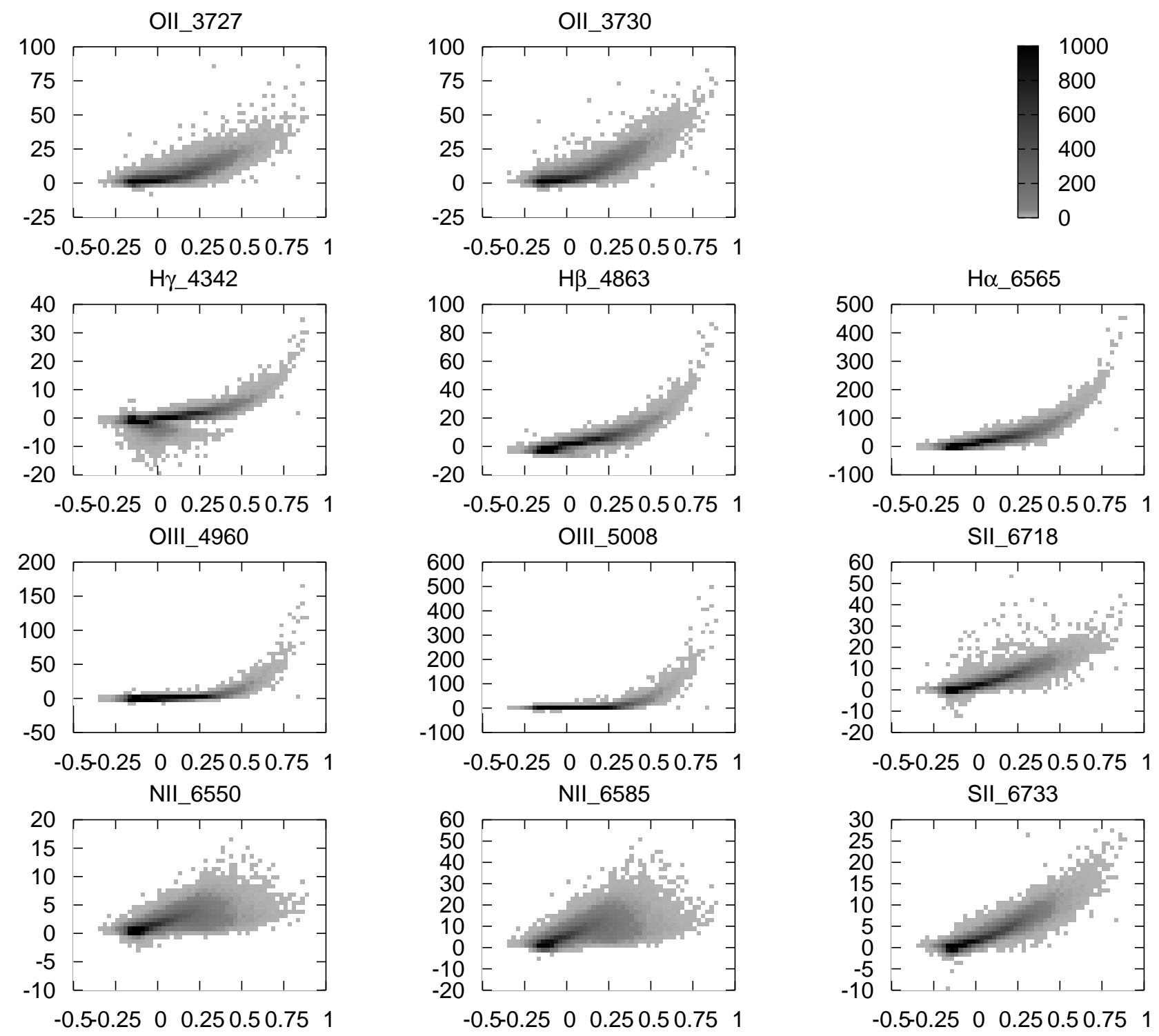

Fig. 2. - EW - eclass distribution of the selected 11 spectral lines. Galaxy counts are plotted in grayscale. The spectral type parameter eclass ( $x$ axis) is small/large for early/late type galaxies. The EWs (in A, $y$ axis) of all lines show a strong type dependence. The absorption dominated early type objects are situated at negative eclass values. At positive eclass the EW's of the emission line galaxies increase with type. 

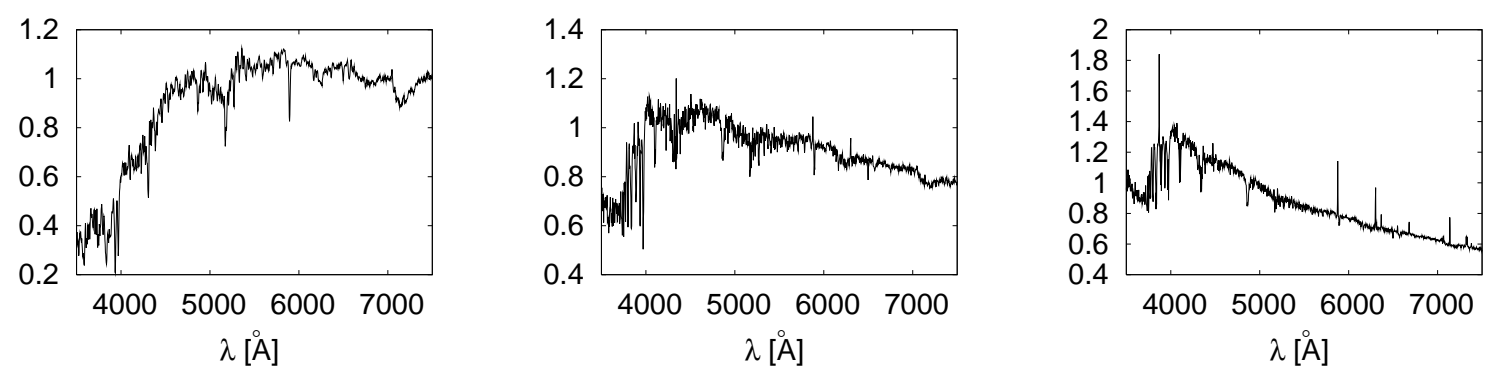

Fig. 3.- Composite galaxy spectra from early type with no emission (left) to emission rich late type (right). 

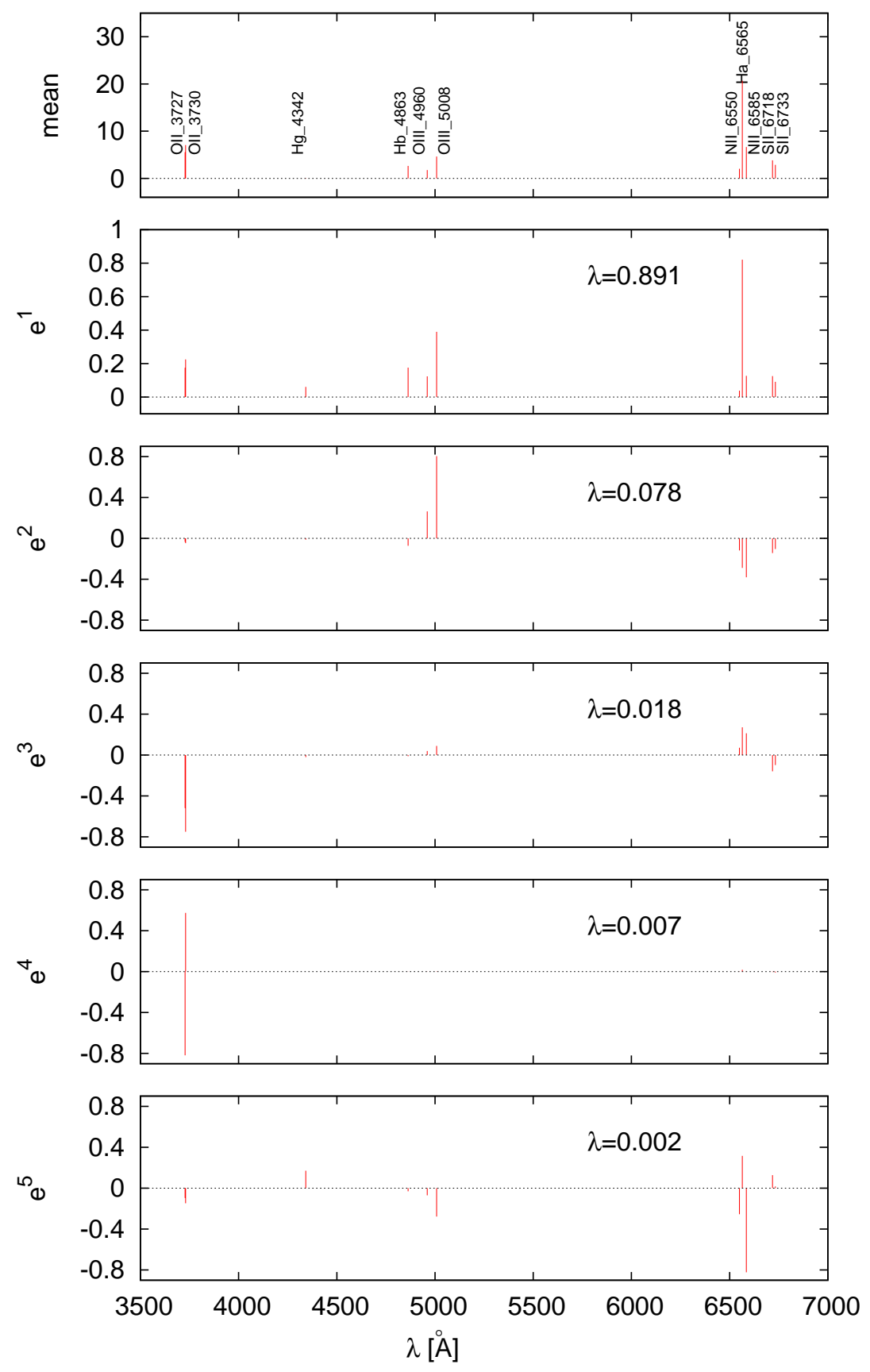

Fig. 4.- Mean vector $\overline{\mathbf{y}}$ and the first five eigenvectors of EW's. For each eigenvector, $\lambda$ denotes the relative information content. See explanation in Section 3.3. 

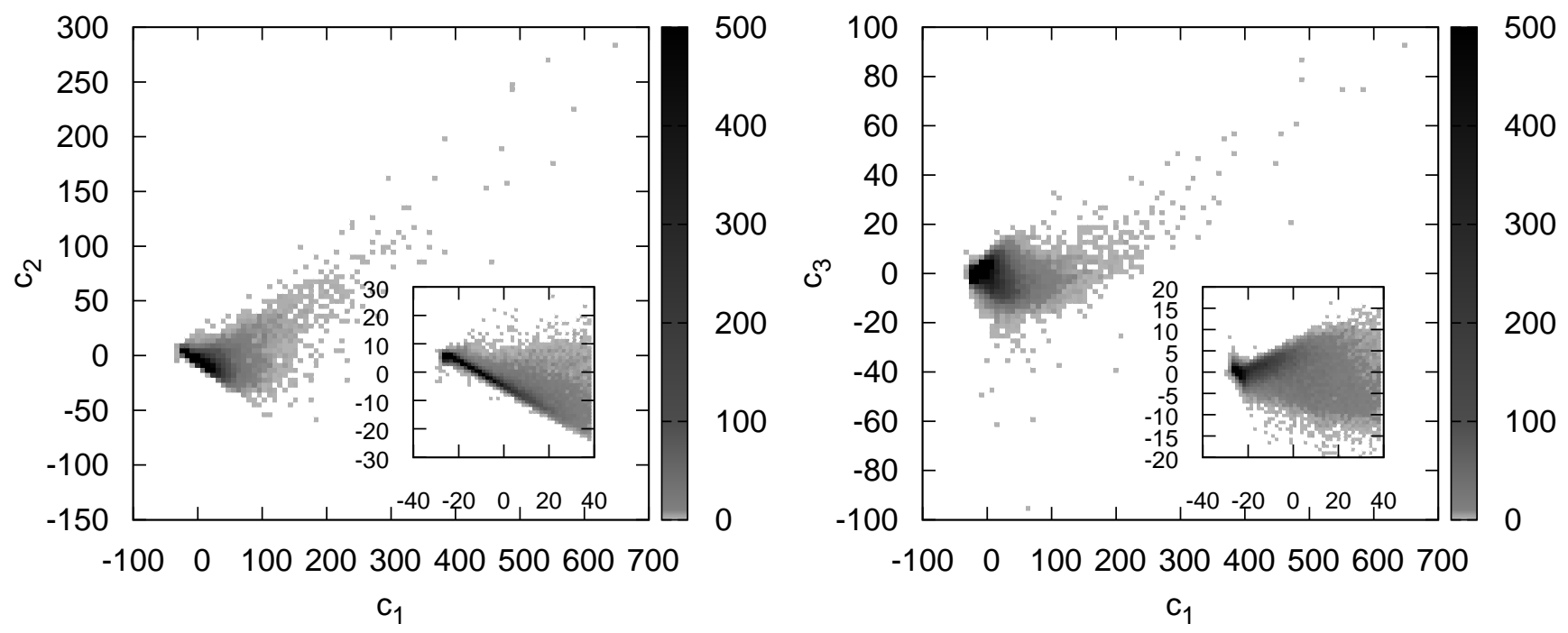

Fig. 5.- Distribution of the emission line galaxies in the subspace of the first and second (left) and the first and the third (right) principal components. PCA shows that the data form a roughly two dimensional manifold in the 11-dimensional EW space. The inset plots show the low $c_{1}$ region zoomed in - the distribution separates into a 'head' and a 'tail'. 


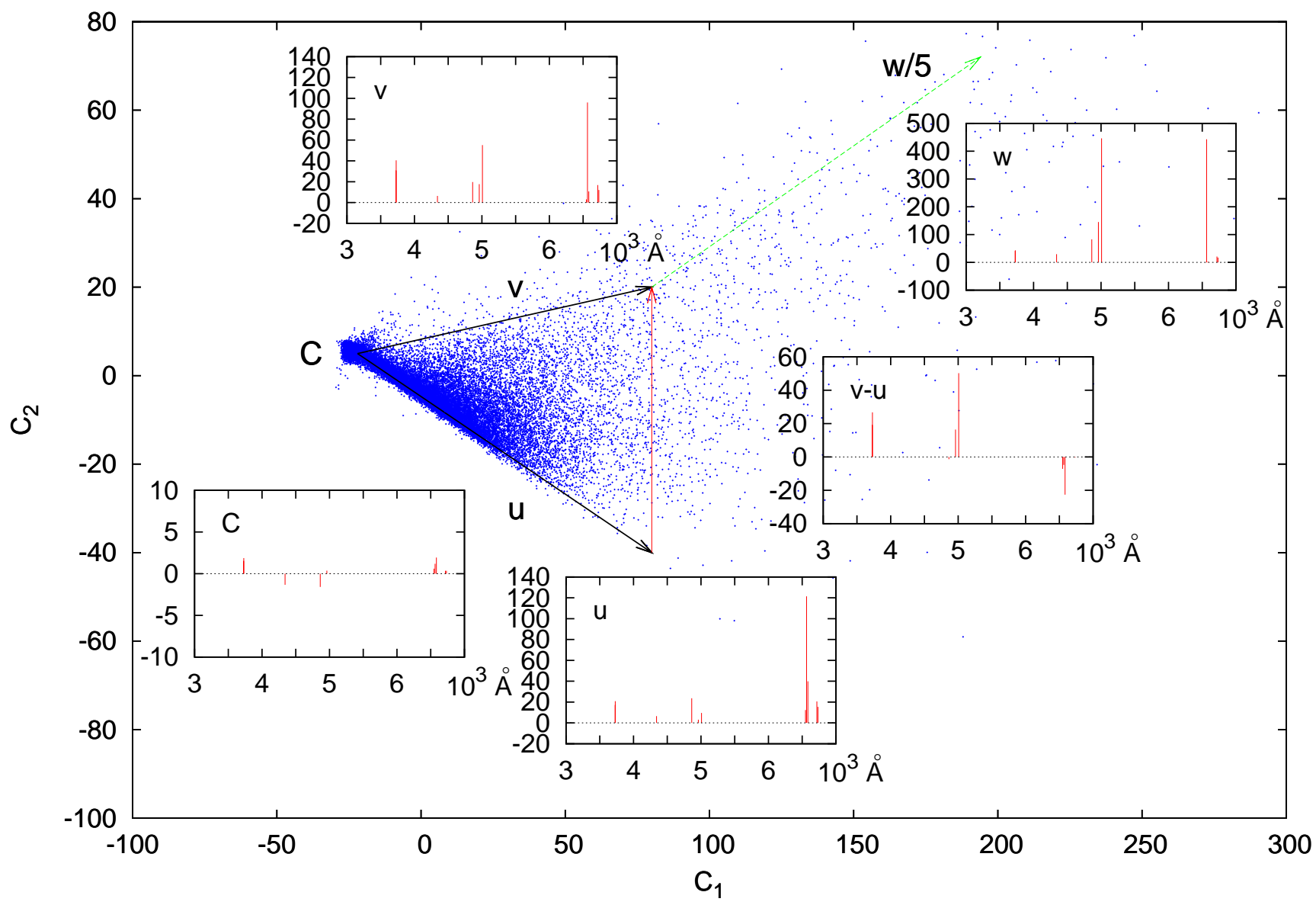

Fig. 6. - EW data projected to the subspace of the first two principal components. Inset plots show 3D-reconstructed EW's corresponding to the origin $C$ and some representative directions. The two vectors $\mathbf{u}$ and $\mathbf{v}$ generate the main locus occupied by the majority of emission line galaxies. Their difference $\mathbf{v}-\mathbf{u}$ contributes to the spectrum in the sense of enhancing oxygen, at the same time depressing nitrogen lines when going in the direction from $\mathbf{u}$ to $\mathbf{v}$. The vector $\mathbf{w}$ together with the previous two vectors generates the strongest emission spectra. It ends out of the range of this figure in the point $E=(500,250,80)$, the shown vector $\mathbf{w} / 5$ has the same direction and fifth the length of $\mathbf{w}$. The spectrum of point $E$ (not shown) is very similar to that of vector w. Very strong Balmer lines and [O III] $\lambda \lambda 4960,5008$, weak [O II] $\lambda \lambda 3727,3730$, as well as nitrogen deficiency can be observed. 


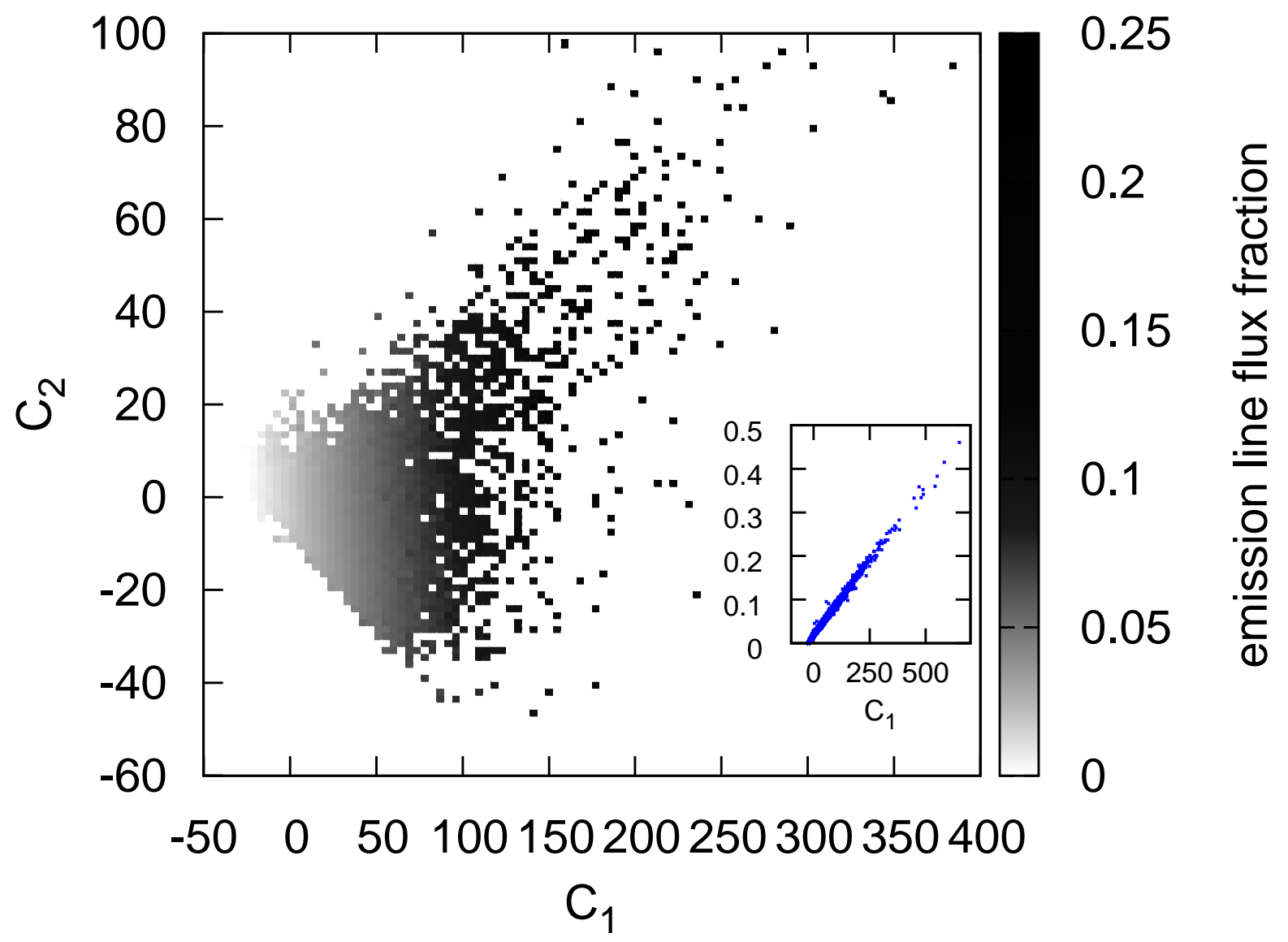

Fig. 7.- Variation of the relative emission line flux $\mu$ averaged over bins (grayscale) shows a linear relation with $c_{1}$ (inset plot). 


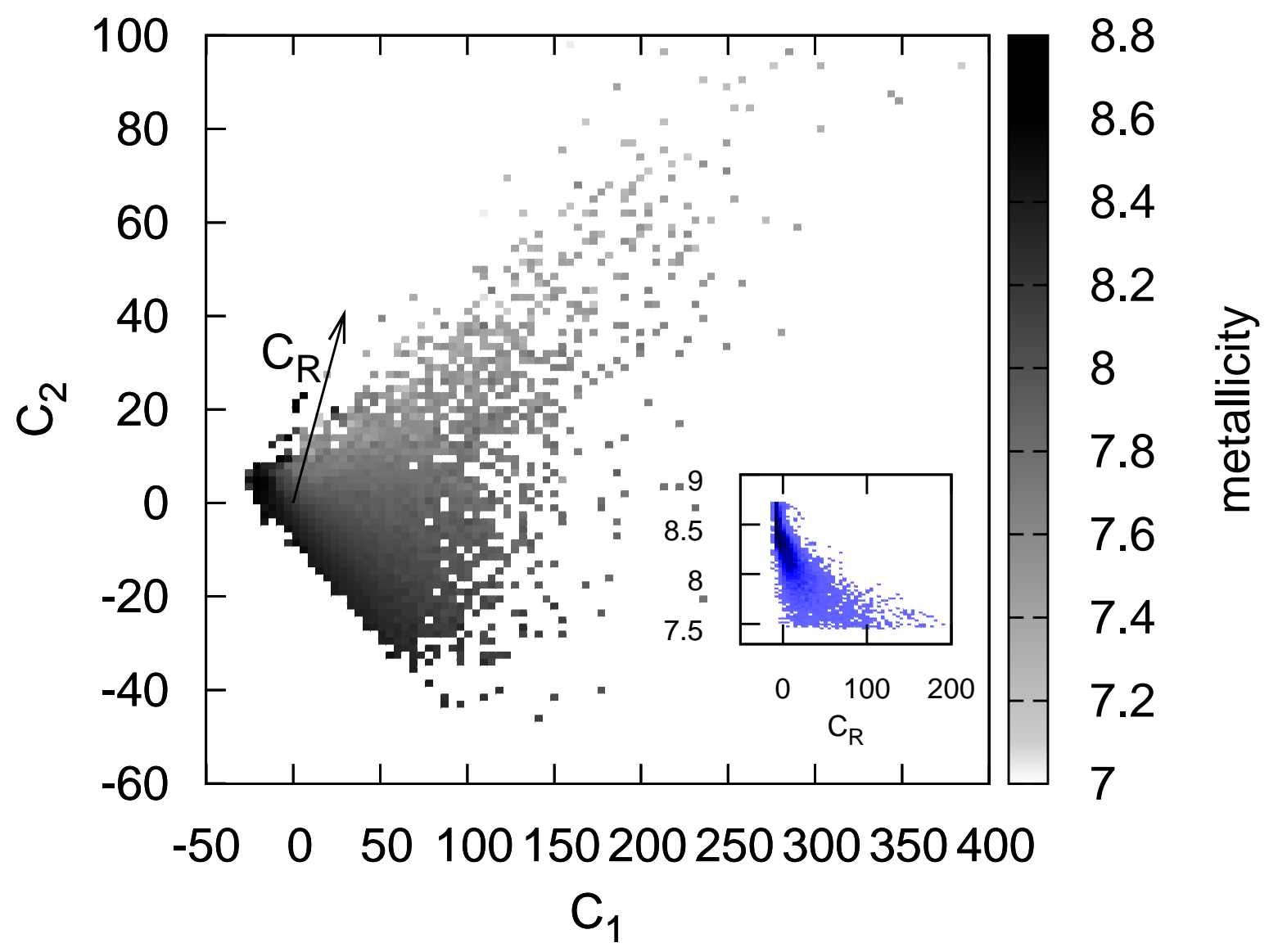

Fig. 8. - Variation of metallicity with the first two PCA coefficients. The quantity $12+\log (\mathrm{O} / \mathrm{H})$ estimated using Equation (4) averaged over bins is plotted in grayscale. The metallicity decreases in the direction of vector $\mathbf{C}_{R}$, which is close to vector $\mathbf{u}-\mathbf{v}$ of Figure 6 . The inset plot shows the distribution of metallicity along $\mathbf{C}_{R}$. 


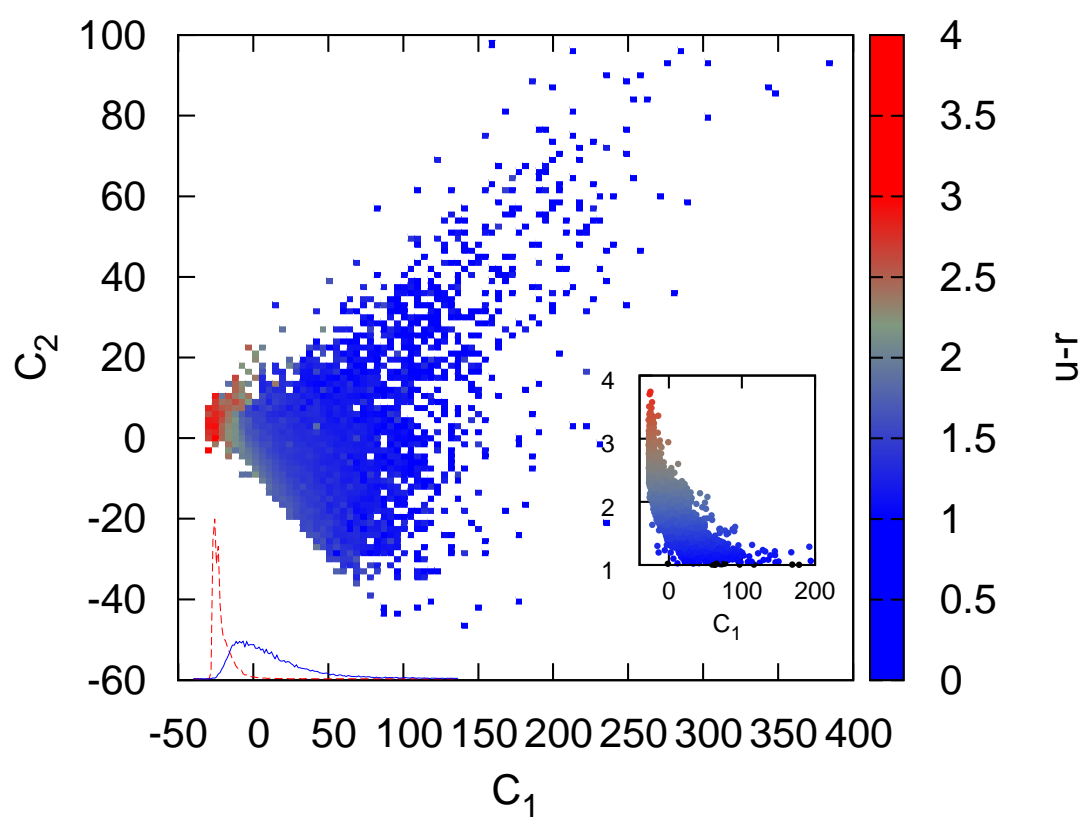

Fig. 9.- Distribution of $u-r$ color in the plane $\left(\mathbf{e}^{1}, \mathbf{e}^{2}\right)$. Main plot: color averaged over pixels ( $u-r$ color-coded, red means $u-r>2.22$, blue $u-r<2.22$, gray is the transition between them). The lowest $c_{1}$ values are dominated by red objects, blue becomes dominant at higher $c_{1}$ values. Transition from red to blue types $(u-r=2.22)$ is around $c_{1}=-20$. Inset plot: $u-r$ vs. $c_{1}$ is a monotonic relation, the higher $c_{1}$, the bluer objects. However, low $c_{1}$ ranges have mixed colors, the relation gets tighter at higher $c_{1}$. Histograms at the bottom: $c_{1}$ distribution of the two color types. Red $(u-r>2.22)$ subset plotted with red dashed line, blue $u-r<2.22$ subset with blue solid line. 


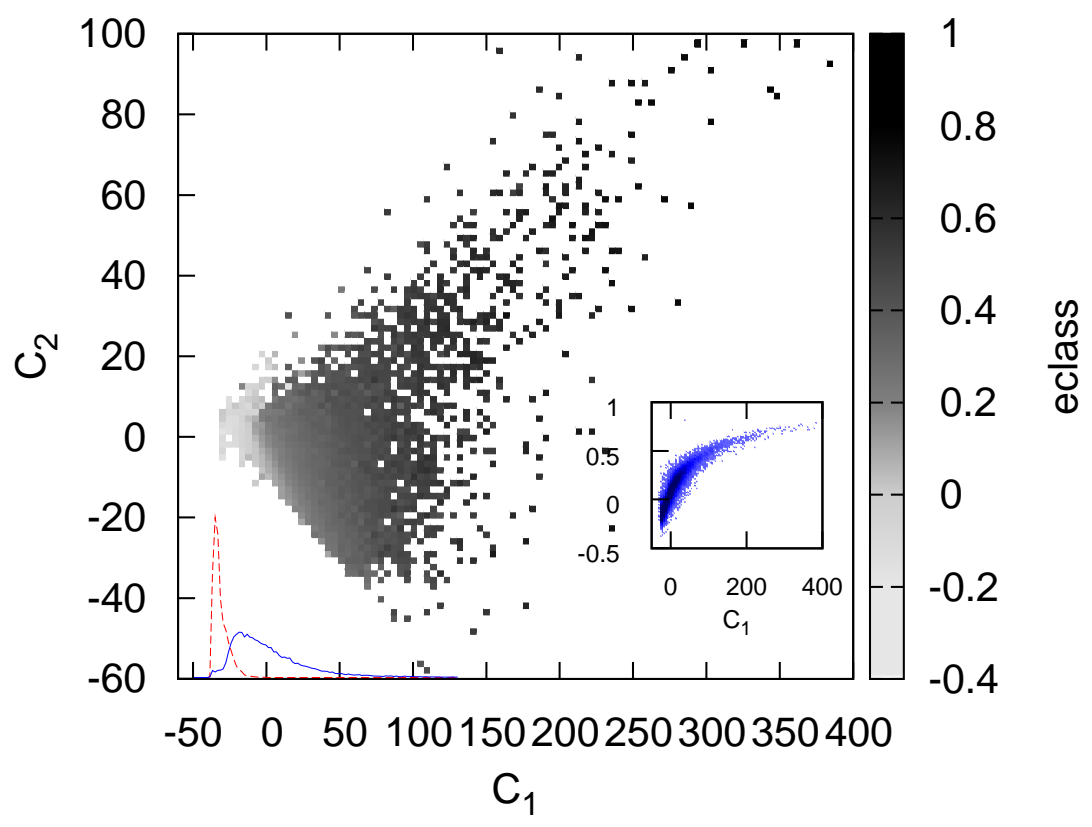

Fig. 10.- Distribution of the spectral type in the plane $\left(\mathbf{e}^{1}, \mathbf{e}^{2}\right)$. Main plot: eclass averaged over pixels. Inset plot: eclass vs. $c_{1}$. We can see the same tendency for eclass as for color in Figure 9. Early types (negative eclass) are at negative $c_{1}$ values, late types (positive eclass) are at higher $c_{1}$. Histograms at the bottom: $c_{1}$ distribution of the two spectral type bins. Red dashed line: eclass $<-0.05$, blue solid line: eclass $>-0.05$. 

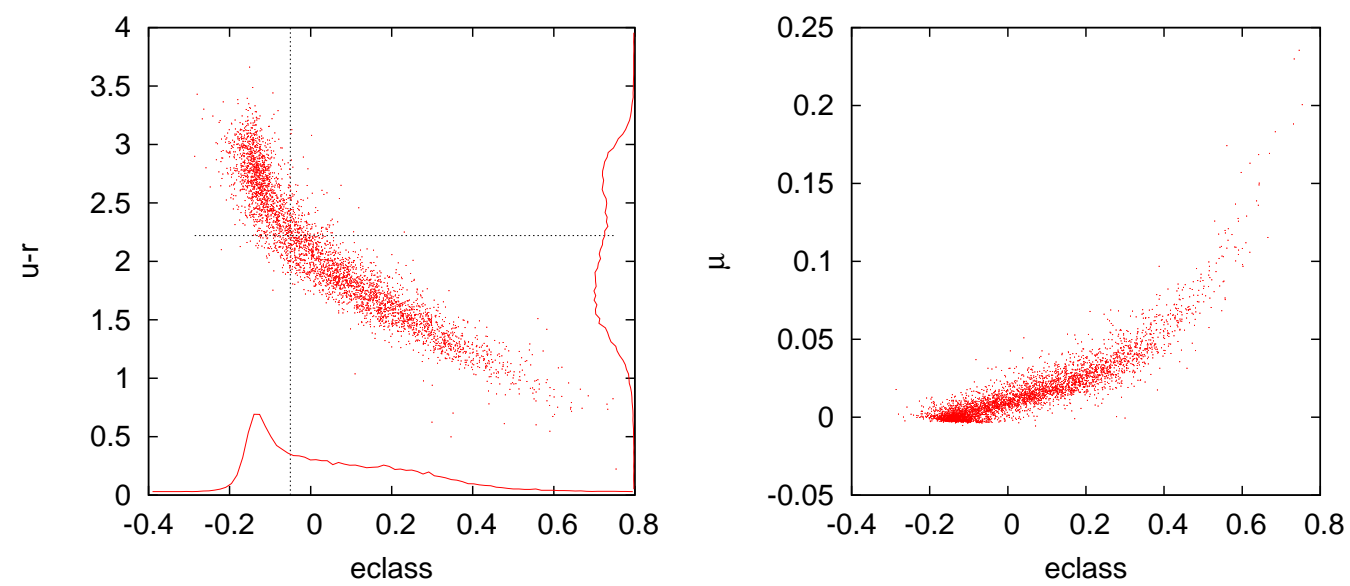

Fig. 11. - Left panel: color $u-r$ vs. spectral type eclass. The relation is nearly linear, apparent bimodality. The $u-r=2.22$ separator of blue and red types (vertical dotted line) corresponds to eclass $\approx=-0.05$ (horizontal dotted line). The histogram of the eclass distribution is plotted on the $x$ axis. The distribution of $u-r$ is projected to the right margin. Right panel: connection between spectral type and relative emission parameter $\mu$. 


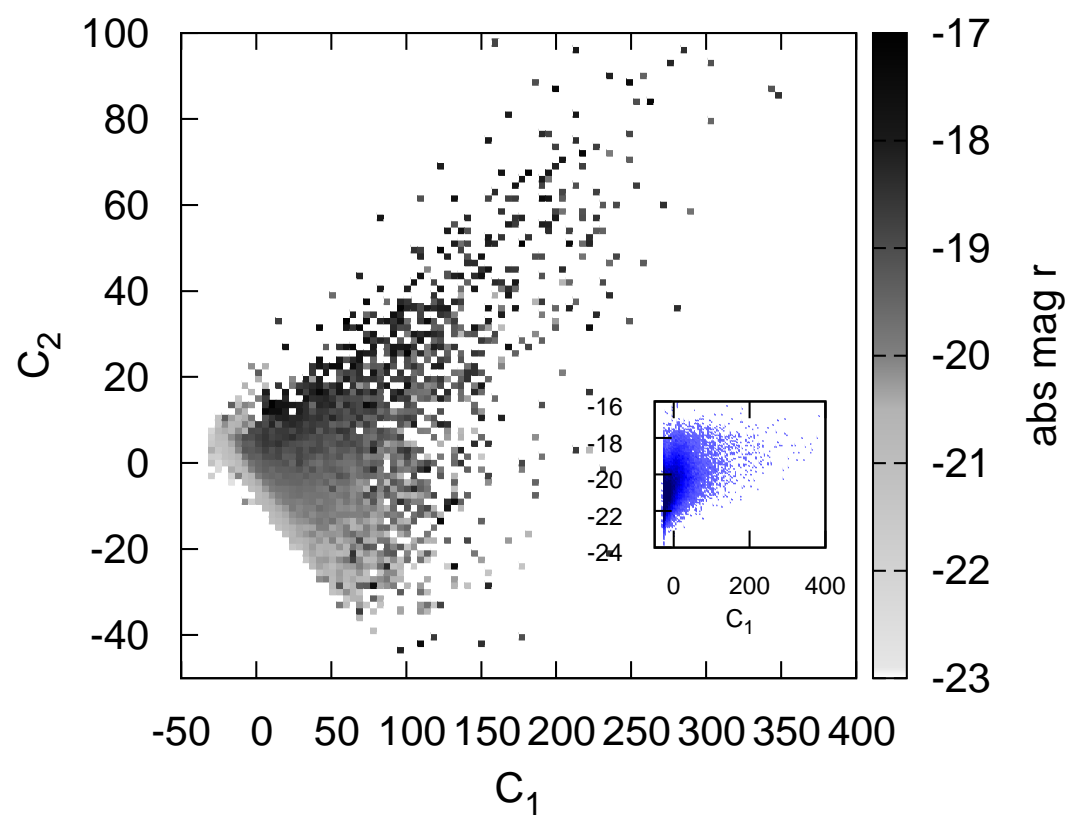

Fig. 12. $-r$-band absolute in the plane of the first two PC's. Main plot: $M_{r}$ averaged over bins (grayscale) in the plane $\left(\mathbf{e}^{1}, \mathbf{e}^{2}\right)$. Inset plot: $M_{r}$ vs. $c_{1}$. On average, luminosity decreases with increasing $c_{1}$, however, the scatter is large. The strongest nebular emission objects are the faintest ones, with $M_{r} \approx-17$. 

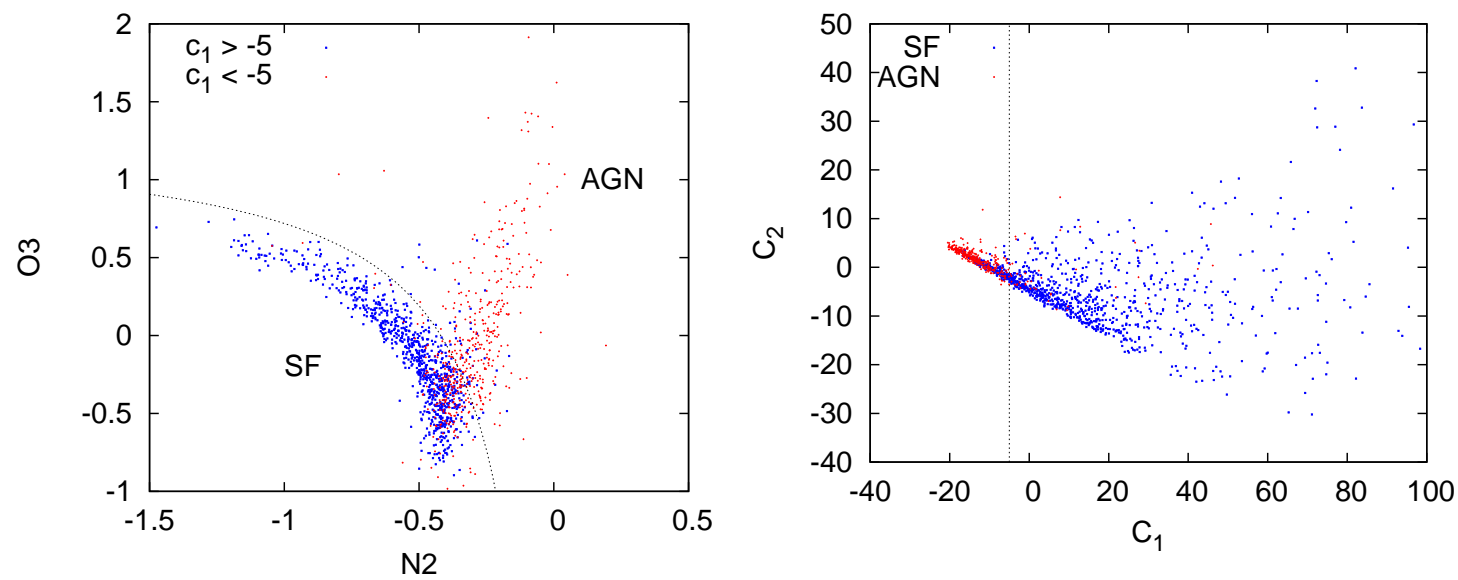

Fig. 13.- Left: N2:O3 diagnostic diagram for distinguishing between star forming galaxies and AGN. The dotted line shows the AGN separator of quation (1). The two types of symbols are selected by $c_{1}=-5$ cut. Righ: AGN (red) and SF (blue) defined by the separator $\mathrm{n}$ the right panel. AGN are situated at $c_{1}<0$. A part of the 'head' is missing because of some negative values among the EW's. In both panels, the scatter plots show a $5 \%$ random subsample.
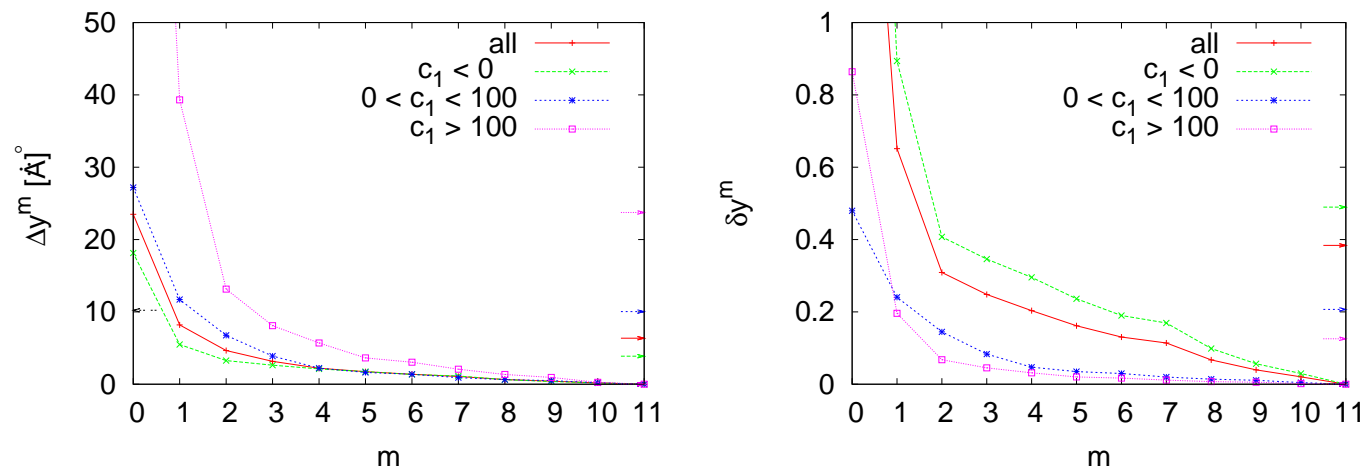

Fig. 14.- Convergence of the spectral line reconstruction. The error of the reconstruction as a function of truncation limit is plotted. $m$ : number of eigencomponents kept; $m=0$ represents the reconstruction using the mean EW for each line, no PCs. Left: EW residuals summed over all lines. Right: relative error. The results are shown for all galaxies (solid thick line) and for three $c_{1}$ bins; larger $c_{1}$ values indicate stronger nebular emission. All EW's can be well reconstructed using the first three eigencomponents. The single arrow at the left margin of the left panel denotes the error of ignoring the emission line flux in eclass $<-0.05$ early type objects, see explanation in the text. The arrows at the right margin show the same quantities for the prediction made from continuum expansion coefficients, see explanation in Section 4. 


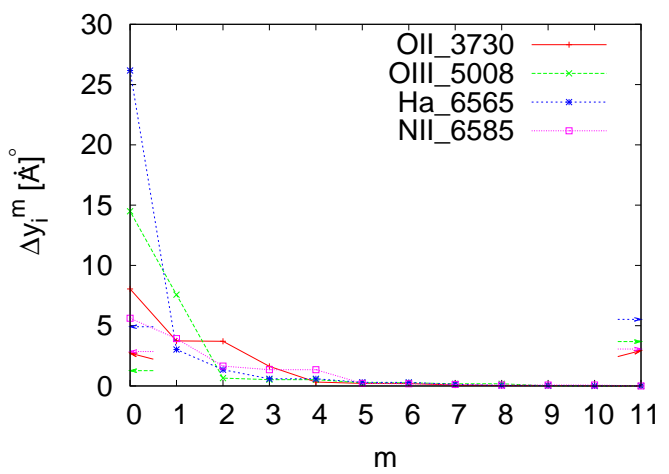

Fig. 15.- Reconstruction of four selected emission lines using $m$ eigencomponents. $\Delta y_{i}$ : sampleaveraged absolute EW error, $m$ : same as in Figure 14. The arrows at the left margin show the error of estimation by zero emission line flux for the eclass $<-0.05$ subset. The arrows at the right margin show the same quantity for the prediction made from continuum expansion coefficients, see explanation in Section 4.

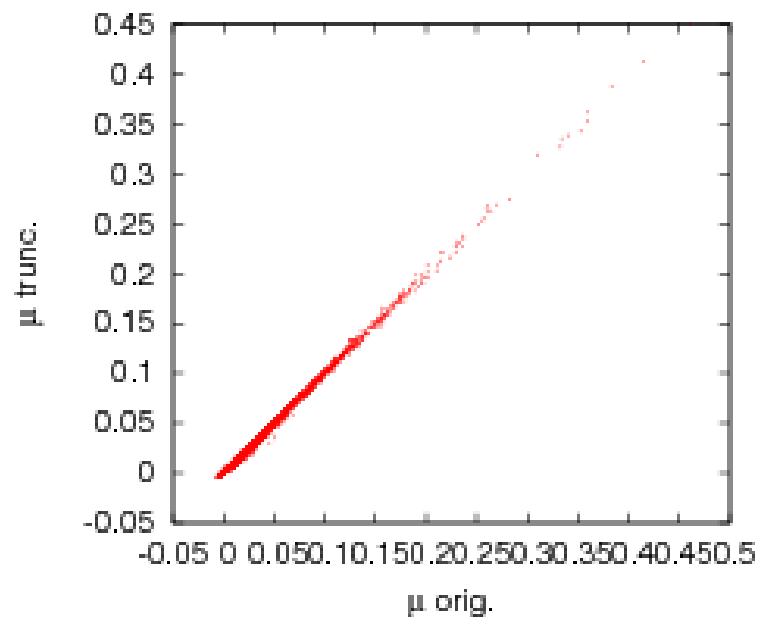

Fig. 16. - Reconstruction of the relative emission strength using truncated eigenbasis. The rms error is 0.001 . 


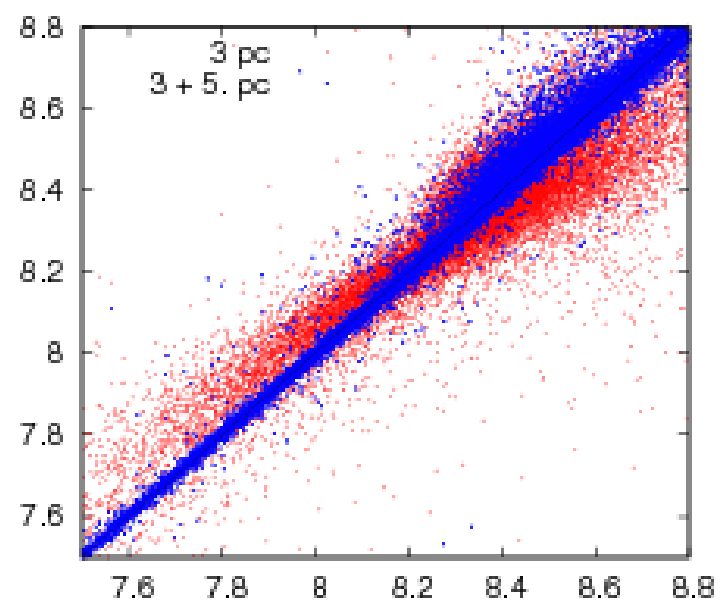

Fig. 17.- Reconstruction of metallicity using truncated eigenbasis. With the first three eigenvectors, the error is relatively large. Adding the fifth eigenvector suppresses the error significantly.

Table 1. AnAlyzed Emission Lines

\begin{tabular}{ll}
\hline \hline \multicolumn{1}{c}{ Line name } & Rest wavelength $[\mathrm{A}]$ \\
\hline$[\mathrm{O}$ II $] \lambda 3727$ & 3727.09 \\
{$[\mathrm{O}$ II $] \lambda 3730$} & 3729.88 \\
$\mathrm{H} \gamma \lambda 4342$ & 4341.68 \\
$\mathrm{H} \beta \lambda 4863$ & 4862.68 \\
{$[\mathrm{O}$ III $] \lambda 4960$} & 4960.29 \\
{$[\mathrm{O}$ III $] \lambda 5008$} & 5008.24 \\
{$[\mathrm{~N}$ II $] \lambda 6550$} & 6549.86 \\
$\mathrm{H} \alpha \lambda 6565$ & 6564.61 \\
{$[\mathrm{~N}$ II $] \lambda 6585$} & 6585.27 \\
{$[\mathrm{~S}$ II $] \lambda 6718$} & 6718.29 \\
{$[\mathrm{~S}$ II $] \lambda 6733$} & 6732.67 \\
\hline
\end{tabular}

Note. - Reference SDSS table, see also SpecLineName table in the catalog science archive. 

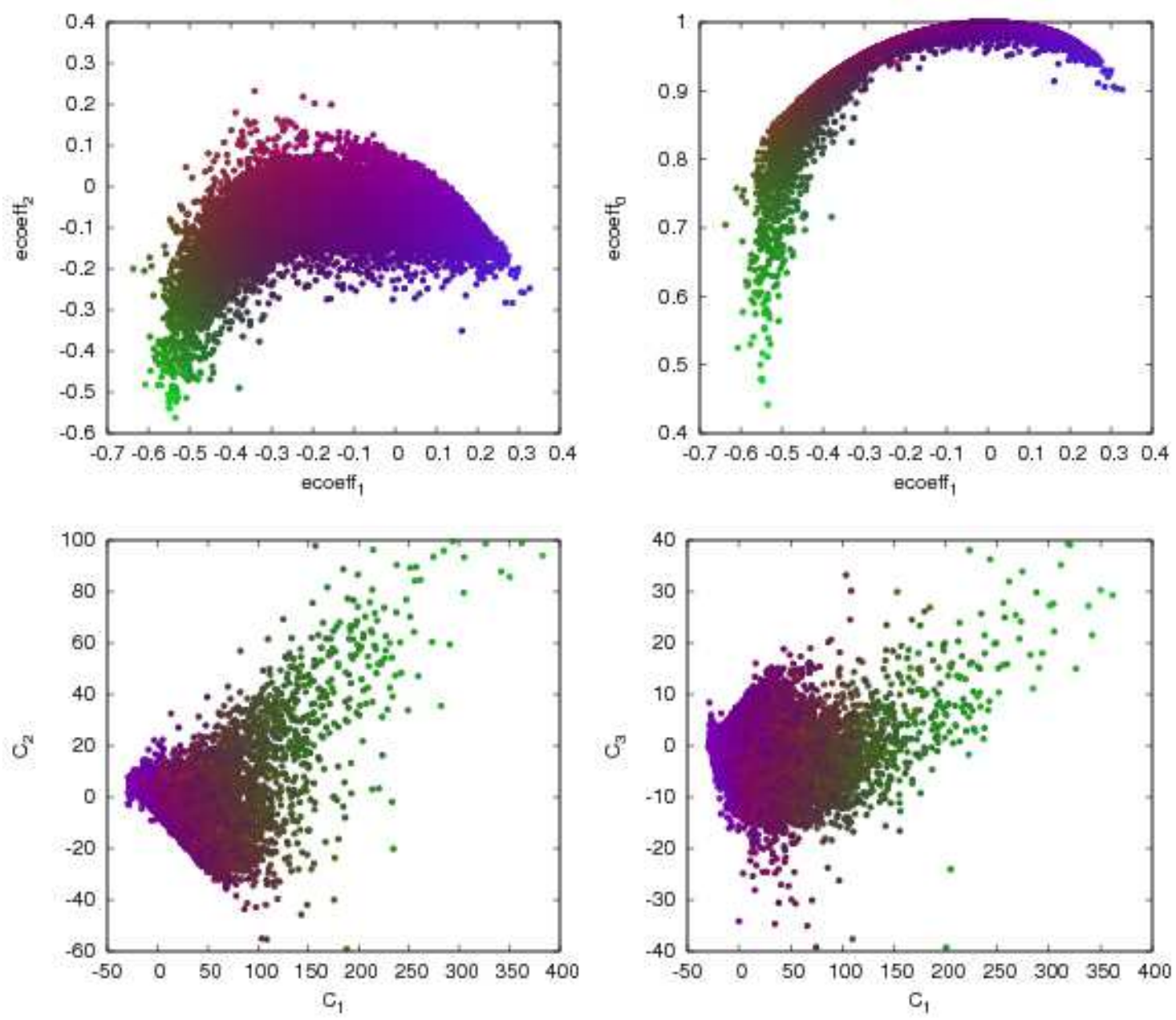

Fig. 18.- Data points plotted in the planes ecoeff :ecoeff $_{2}$ (top left), ecoeff eecoeff $_{3}$ (top right), $c_{1}: c_{2}$ (bottom left) and $c_{1}: c_{3}$ (bottom left). The coloring is made by rgb-coding of ecoeff $f_{1}$ (green), ecoeff $f_{2}$ (blue), ecoeff $f_{3}$ (red). The same coloring is applied to the emission line PCA subspaces. By matching the points of the same colors in the various plots we can see how the ecoeff $f_{i}$ regions are mapped into the $c_{i}$ space. The image shows, that (at least for ecoeff $f_{1}<0.1$, which means positive eclass, later type objects) there is a mapping. Early types are not resolved, however, they are located in the 'head' of the distribution, having weak emission lines. 

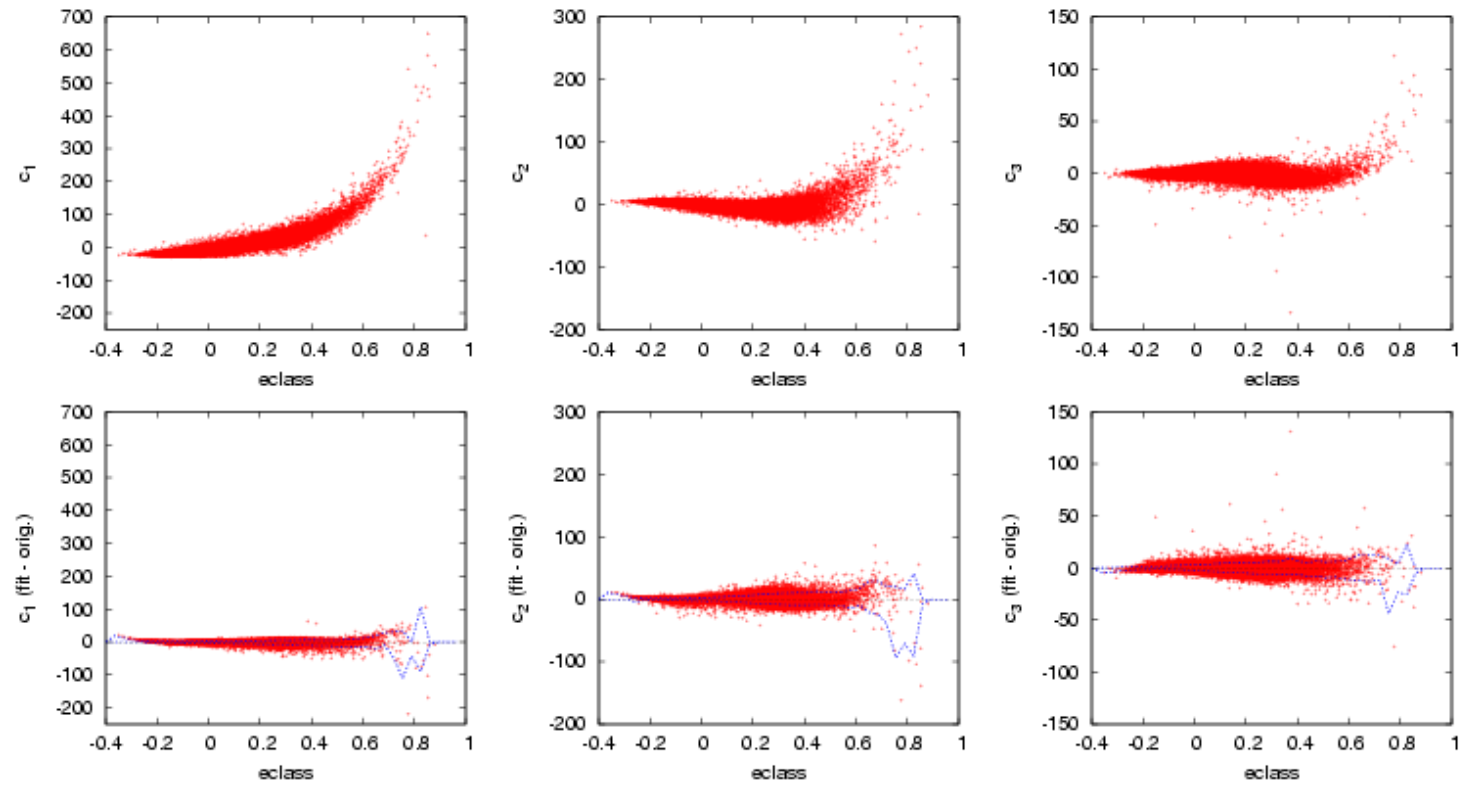

Fig. 19.- Top: PC coefficients as a function of eclass. Bottom: the residuals of the first three emission line PC coefficients after subtracting the continuum fit, as a function of the continuum type ecoeff. An empirical connection between the continuum spectral shape and the nebular emission pattern can be established from the observed correlations. The average residual scatter after subtracting the fitted estimator is plotted with blue dotted lines as a function of the spectral type. 

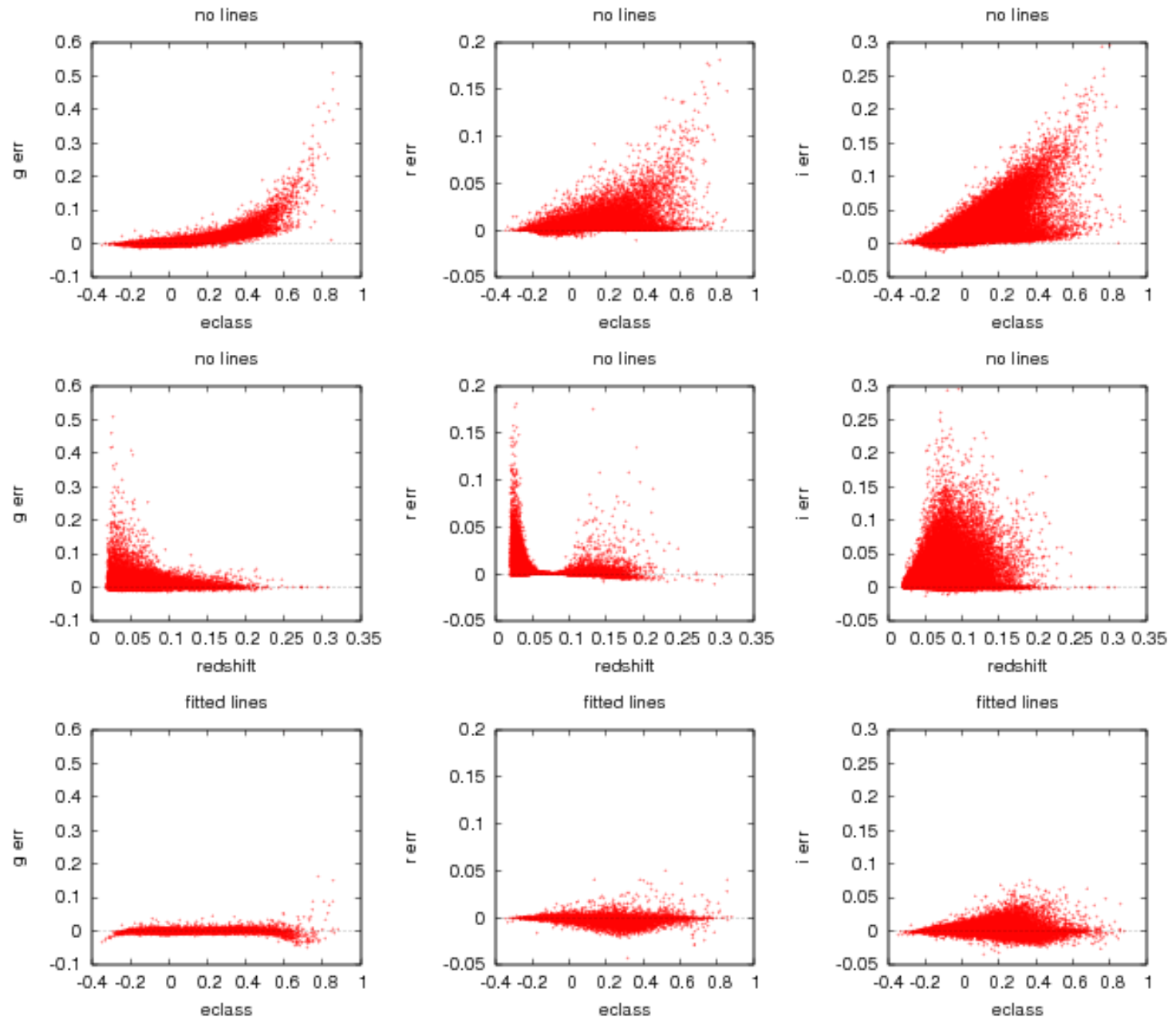

Fig. 20.- Difference in $g, r$ and $i$ magnitude, if the photometry is made without emission lines, as a function of spectral type (first row) and redshift (second row). Late spectral types at larger eclass having stronger nebular emission will have larger errors. Redshift dependence shows pattern caused by lines being redshifted into and out from the filter. Third row: magnitude errors of the simulated photometry with emission line from the continuum fit, as a function of spectral type. 
Table 2. The First Five EIGENVECtors

\begin{tabular}{lccccc}
\hline \hline Line name & $\begin{array}{c}e_{1} \\
(0.891)\end{array}$ & $\begin{array}{c}e_{2} \\
(0.078)\end{array}$ & $\begin{array}{c}e_{3} \\
(0.018)\end{array}$ & $\begin{array}{c}e_{4} \\
(0.007)\end{array}$ & $\begin{array}{c}e_{5} \\
(0.002)\end{array}$ \\
\hline$[\mathrm{O}$ II $] \lambda 3727$ & 0.176 & -0.033 & -0.518 & -0.819 & -0.095 \\
{$[\mathrm{O}$ II $] \lambda 3730$} & 0.225 & -0.046 & -0.750 & 0.574 & -0.148 \\
$\mathrm{H} \gamma \lambda 4342$ & 0.061 & -0.012 & -0.021 & 0.000 & 0.171 \\
$\mathrm{H} \beta \lambda 4863$ & 0.176 & -0.072 & -0.012 & 0.001 & -0.031 \\
{$[\mathrm{O}$ III $] \lambda 4960$} & 0.124 & 0.265 & 0.040 & 0.001 & -0.070 \\
{$[\mathrm{O}$ III $] \lambda 5008$} & 0.390 & 0.805 & 0.089 & 0.002 & -0.278 \\
{$[\mathrm{~N}$ II $] \lambda 6550$} & 0.039 & -0.118 & 0.072 & -0.001 & -0.254 \\
$\mathrm{H} \alpha \lambda 6565$ & 0.821 & -0.289 & 0.271 & 0.018 & 0.315 \\
{$[\mathrm{~N}$ II $] \lambda 6585$} & 0.127 & -0.380 & 0.213 & -0.003 & -0.822 \\
{$[\mathrm{~S} \mathrm{II}] \lambda 6718$} & 0.125 & -0.143 & -0.159 & -0.004 & 0.127 \\
{$[\mathrm{~S}$ II $] \lambda 6733$} & 0.091 & -0.104 & -0.098 & -0.008 & 0.017 \\
\hline
\end{tabular}

Note. - The first five EW principal components ordered by their relative information content. The eigenvalue of each eigenvector is given in round brackets in the column header. 
Table 3. The Fit Polynom COEFficients of $c_{i}$

\begin{tabular}{lrrr}
\hline \hline & $c_{1}$ & $c_{2}$ & $c_{3}$ \\
\hline$\alpha$ & 1657.808 & 674.528 & 508.194 \\
$\beta_{0}$ & -4390.826 & -191.740 & -639.650 \\
$\beta_{1}$ & 488.087 & 108.530 & -27.376 \\
$\beta_{2}$ & -31.382 & -150.234 & -16.725 \\
$\gamma_{00}$ & 2717.923 & -481.908 & 132.141 \\
$\gamma_{01}$ & -610.956 & -59.598 & 25.392 \\
$\gamma_{02}$ & -153.612 & 226.695 & -2.644 \\
$\gamma_{11}$ & 546.841 & -498.071 & -245.997 \\
$\gamma_{12}$ & 212.847 & 82.486 & -36.546 \\
$\gamma_{22}$ & 433.339 & -617.698 & -375.677 \\
\hline
\end{tabular}

Note. - Fitted polynom coefficients of equation (8) for $c_{1}, c_{2}$ and $c_{3}$ as a function of ecoeff . $_{i}$

Table 4. The Fit Polynom COEFficients of SELECTED LineS

\begin{tabular}{lrrrr}
\hline \hline & $\mathrm{H} \alpha$ & {$[\mathrm{O}$ III $]$} & {$[\mathrm{O}$ II $]$} & {$[\mathrm{N}$ II $]$} \\
\hline$\alpha$ & 1178.370503 & 2655.215341 & 308.461569 & -61.094836 \\
$\beta_{0}$ & -3486.511250 & -4071.111890 & -1406.454947 & -443.963678 \\
$\beta_{1}$ & 281.981021 & 1477.873084 & 625.325629 & -215.602859 \\
$\beta_{2}$ & -100.191558 & -36.292149 & 38.422858 & -15.750437 \\
$\gamma_{00}$ & 2315.964983 & 1415.882134 & 1103.062075 & 510.520196 \\
$\gamma_{01}$ & -398.852773 & -1443.357079 & -664.083788 & 153.924612 \\
$\gamma_{02}$ & -86.340819 & 40.335051 & -90.761428 & -77.564287 \\
$\gamma_{11}$ & 550.149412 & -229.362689 & 579.736300 & 149.155350 \\
$\gamma_{12}$ & 80.078273 & 309.940485 & 143.727637 & -85.912611 \\
$\gamma_{22}$ & 454.181171 & -646.157142 & 663.971699 & 210.729024 \\
\hline
\end{tabular}

Note. - Fitted polynom coefficients of equation (8) for $\mathrm{H} \alpha$, [O III], $[\mathrm{OII}]$ and $[\mathrm{NII}]$ as a function of ecoeff . $_{i}$ 
Table 5. The RMS ERror OF PHOTO-Z

\begin{tabular}{llll}
\hline \hline & \multicolumn{1}{c}{$\mathrm{t}$} & original & added lines \\
\hline all & $0-55$ & 0.0694 & 0.0680 \\
red & $0-20$ & 0.0557 & 0.0556 \\
blue & $21-55$ & 0.0876 & 0.0843 \\
bluest & $50-55$ & 0.1512 & 0.1374 \\
\hline
\end{tabular}

Note. - The rms error of photo-z for different galaxy types, for two different templates sets. Column 3 lists the error of the redshift estimate when the original CWW templates are trained and used as templates. Column 4 shows the results in the case when the emission lines are replaced before the training. The type parameter $t$ goes from 0 to 55, 0 being the reddest, 55 the bluest type. 